CERN-LHC/98-6 (DLO)

6 September 1998

\title{
FEATURES AND TECHNOLOGY OF FERROELECTRIC ELECTRON EMISSION
}

\author{
H. Riege \\ CERN LHC-Division, CH-1211 Geneva 23, Switzerland \\ I. Boscolo \\ Department of Physics, University of Milan, I-20133 Milan, Italy \\ J. Handerek \\ University of Silesia, Institute of Physics, P-40007 Katowice, Poland \\ U. Herleb \\ Institute of Physics, University of Erlangen-Nürnberg, D-91058 Erlangen, Germany
}

\begin{abstract}
Spontaneous electrical polarization of ferroelectric materials can be changed either by reversal or by phase transition from a ferroelectric into a non-polar state or vice versa. If spontaneous polarization changes are induced at a submicrosecond time-scale, strong uncompensated surface charge densities and related fields are generated, which may lead to the intense self-emission of electrons from the negatively-charged free surface areas of the ferroelectric cathode. The nature of this self-emission differs essentially from other methods of ferroelectric electron emission and from conventional electron emission in that the latter methods are only achieved by extracting electrons with externally applied electric fields. When electron guns are constructed with ferroelectric cathodes, new design criteria have to be taken into account. The intensity, the energy, the temporal and spatial distribution and the repetition rate of the emitted electron beams can be adjusted within wide limits. The advantages of ferroelectric cathodes and the technological difficulties arising during their production, preparation, and operation are identified and discussed and solutions to the problems are proposed. Experiences with a few applications of ferroelectric electron emission are reported and suggestions for further applications are made.
\end{abstract}



INTRODUCTION

Electron emission during the change of spontaneous polarization of ferroelectric (FE) material was noted by Miller and Savage in 1960 [1]. Early experimental observations of weak electron emission from ferroelectrics were, for example, reported in Refs. [2, 3]. 'Strong electron emission' of more than $100 \mathrm{~A} / \mathrm{cm}^{2}$ from FE ceramics was detected at CERN in 1988 [4-13]. Later on different methods were studied in other laboratories to extract intense electron beams from FE cathodes (for example, Refs. [14-22]), and confusion arose about what is meant by 'ferroelectric electron emission'. The phenomenological appearance and some of the basic processes differ in these latter cases from those in the original 'strong FE emission mode' [4-13]. The authors feel obliged to clarify in this paper, through the use of a simple model and experimental results, what they mean by 'FE emission', and to point out some of its advantages and differences compared with other modes. In previous papers [5-13] attempts were made to discuss the principles, processes and apparent features of typical FE emission, the observations made experimentally, and the performance of FE cathodes in several applications. This paper will also deal with some of the technological aspects and difficulties arising when designing, fabricating and operating FE cathodes and FE electron guns for a dedicated purpose. The intrinsic features of FE emission differ so significantly from classical electron emission methods that new designcriteria for electron guns with FE cathodes have been introduced. Unlike classical emission methods FE emission does not need an externally applied extraction field, since the field emission of the electrons is achieved by the spontaneous polarization fields of the FE materzial itself. FE cathodes and FE electron guns offer many advantages over classical electron sources. However, at present new technological tools and methods still have to be developed and optimized for each specific application.

\section{PHENOMENOLOGICAL DESCRIPTION OF FERROELECTRIC ELECTRON EMISSION}

\subsection{Spontaneous polarization switching and electron emission}

The mode of strong FE electron emission is defined by means of a simple model (Fig. 1) and compared with other modes of FE electron emission. Phenomenological descriptions of its features are given, taking into account the experimental and operational results from FE cathodes obtained so far by the authors and backed up by results published elsewhere. The description of the FE emission phenomena in terms of microscopic processes and of FE physics interpretations is restricted to a minimum in this paper and will be dealt with in a future article.

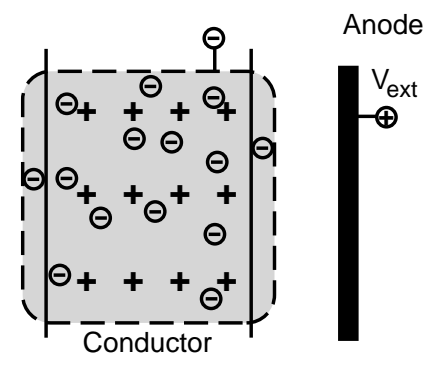

a)

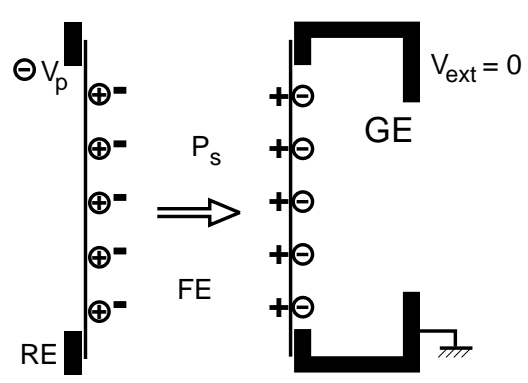

b)

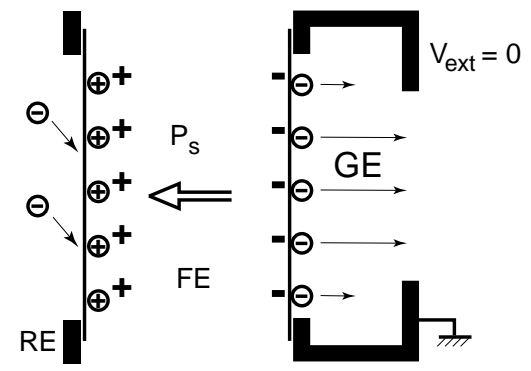

c)

FIG. 1. a) Conventional extraction of electrons from a neutral, conductive emitter, b) charge-compensated FE sample, and c) electron emission after polarization change. $P_{s}=$ spontaneous polarization; $V_{p}=$ excitation pulse voltage; $\mathrm{V}_{\text {ext }}\left(\mathrm{E}_{\mathrm{ext}}\right)=$ extraction potential (field). 
Conventional types of electron emission, for example field emission, thermionic emission, secondary emission or photoemission, are characterized by the extraction of electrons from a quasi-neutral, predominantly conducting piece of matter by means of an external electric field (Fig. 1a). A spontaneously polarized, but originally surface-charge-compensated FE sample (Fig. 1b) is transformed (switched) by a rapid change $\Delta \mathrm{P}_{\mathrm{s}}$ (e.g. by reversal, creation or annihilation of spontaneous polarization $\mathrm{P}_{\mathrm{s}}$ ) into an object resembling a charged capacitor with a resultant surface charge density equal to $\Delta \mathrm{P}_{\mathrm{s}}($ Fig. 1c). This surface charge generates a surface potential difference,

$$
\Delta \mathrm{V}_{\mathrm{s}}=\Delta \mathrm{P}_{\mathrm{s}} \mathrm{d} / \varepsilon
$$

across the FE sample, where $\varepsilon$ is the dielectric constant and $d$ the thickness of the sample. The change $\Delta \mathrm{P}_{\mathrm{s}}$ can be induced by applying a rise of temperature (for example, $[2-4,6]$ ), a mechanical pressure pulse [23], an intense laser pulse [9, 10, 12] or, as first reported by Rosenmann et al. [3], a high-voltage (HV) pulse via surface electrodes [5-8, 10, 11]. The faster the surface-charge density $\mathrm{q}_{\mathrm{s}}$ builds up during all types of excitation the higher is the resulting maximum current density $\Delta \mathrm{j}_{\max }$ :

$$
\Delta \mathrm{j}_{\max } \propto \mathrm{dP}_{\mathrm{s}} / \mathrm{dt},
$$

since the charge compensating processes cannot follow quickly enough the $\mathrm{P}_{\mathrm{s}}$-change. The resultant surface field caused by the induced surface-charge density may become so strong that electrons are expelled from the negatively charged FE surface without an additionally applied external field. Figures 1a and 1c demonstrate that this type of emission from FE (sometimes called 'self-emission' in this paper) is in contrast to conventional electron extraction from a neutral cathode. In order to achieve FE emission with electric field excitation an HV pulse of negative polarity with respect to the emitting grid surface (GE) must therefore be applied to the opposite (rear) surface electrode (RE) of the FE disc. The switching field is then directed from GE to RE. Figure 2a shows typical charge and current waveforms of electrons emitted without an extraction field from an FE cathode switched by an HV pulse generated in the electrical circuit shown in Fig. 2b. The order of magnitude of the emitted charge and current values, which are quantitatively measured, depends strongly on the mechanical layout and on the electrical parameters of the emission current circuit. At very low switching rates, for example with $50-\mathrm{Hz}$ sine-wave excitation, no emission is expected according to Eq. (2) assuming homogeneous switching throughout the FE sample. However, in spite of the very small number of FE domains switching per unit time, weak electron emission is observed (for example $[3,5,24,25])$ because the inhomogeneous, burst-like, 'full' $\mathrm{P}_{\mathrm{s}}$-switching of groups of domains generates locally the high field amplitudes on the surface necessary for electron emission. The electrons are emitted from the FE surface into the adjacent vacuum or gas-filled volume with a distribution of finite kinetic electron energies:

$$
\mathrm{E}_{\mathrm{kin}}=\mathrm{e} \Delta \mathrm{V}_{\mathrm{s}} \text {. }
$$

The maximum kinetic energies may exceed $100 \mathrm{keV}$ as indicated by Eq. (1) and as, for example, reported in Refs. [24, 25]. It is, hence, physically meaningless to distinguish between a 'low and a high emission current regime' [22], since, in agreement with observation, the same laws of nature govern electrodynamically the emission processes for low and high intensities. 
a)

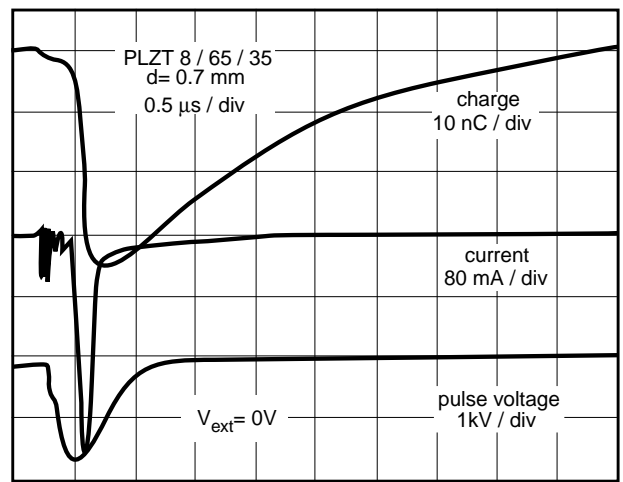

b)

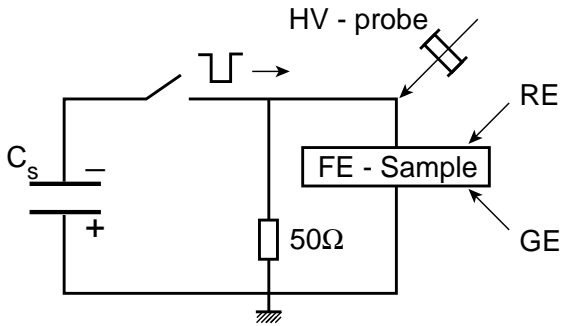

FIG. 2. a) Charge and current waveforms of self-emitted electrons after HV pulse switching from an FE cathode with the switching field directed from the GE to RE surface and with an amplitude well above the threshold field (500 ns/div). b) Basic electrical circuit.

\subsection{Electric-field-excited FE electron emission}

$\mathrm{P}_{\mathrm{s}}$-switching is excited by a negative HV pulse applied to a contiguous RE electrode and, above a characteristic threshold of the excitation field, FE electron emission occurs from the bare FE surface areas of a gridded ('semi-transparent') electrode on the GE surface.

$\mathrm{HV}$ pulsing of an un-poled FE sample with reversed field direction from RE to GE via surface electrodes, as described in Refs. [14-22], initiates $\mathrm{P}_{\mathrm{s}}$-switching at the same threshold field level. However, the sequence of processes following the switching, including plasma formation and electron extraction by external field, may widely differ from the FE self-emission described above. When the reverse field excitation is employed, the free FE surface on the GE becomes positively charged after $\mathrm{P}_{\mathrm{s}}$-switching during the rising part of the HV pulse. Therefore, at this moment, electrons are emitted from the negatively charged metallic grid towards the positively charged bare FE surface parts and can only be extracted into the vacuum gap by means of an external extraction potential $\mathrm{V}_{\mathrm{ext}}$. If the polarization relaxes back during the falling part of the HV pulse or afterwards, a similar situation arises as with the FE 'selfemission' induced directly during the rise time of the HV pulse. In both cases electrons are expelled by the negative potential generated on the bare FE surface. However, the emission intensities and the kinetic electron energies are differently influenced in both cases by the cathode surface potential distributions and by the plasma, which had formed earlier by $\mathrm{P}_{\mathrm{s}}$ switching during the rise time of the excitation pulse. Figure 3 shows typical emitted charge

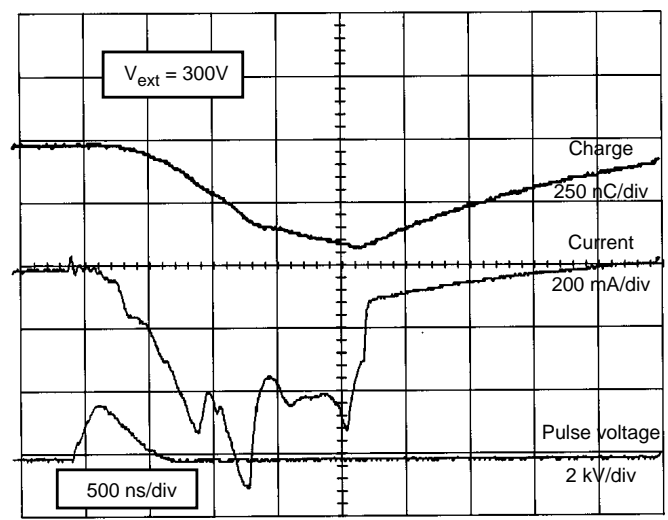

FIG. 3. Emitted charge (top), current (middle) and HV waveforms (bottom) measured under electric field excitation with field direction from RE to GE and under the influence of an external extraction potential $\mathrm{V}_{\text {ext }}$ [21]. 
and current waveforms observed with FE cathodes under a finite extraction potential and an $\mathrm{HV}$-pulse-excited $\mathrm{P}_{\mathrm{s}}$-switching with reverse field direction compared to the FE self-emission mode (Fig. 2a). The emitted current waveforms are more complex than the waveforms observed under FE self-emission conditions and may extend over intervals of several $\mu \mathrm{s.}$

The consequences of $\mathrm{P}_{\mathrm{s}}$-switching can be observed via the light emitted from the $\mathrm{FE}$ cathode surface when using a fast optical framing camera of submicrosecond time-resolution $(\approx$ $50 \mathrm{~ns}$ in this case). Whenever the switching threshold is reached, intense light emission starts either from the bare FE surface areas of the GE (Fig. 4a) in real FE emission mode (Fig. 4b), or mainly from the metallic parts of the GE in reverse-field excitation mode (Fig. 4c). The emitted electron beam can be observed with a luminescent screen. The case of FE self-emission without extraction field and without beam focusing is shown in Fig. 4d. By means of a cylindrical, electrostatic lens following the FE cathode and charged with a negative potential of $-2 \mathrm{kV}$ and by applying a post-accelerating potential of $+1 \mathrm{kV}$ to a gap behind this lens, individual beams starting from separate bare FE cathode surface areas are focused onto the screen (Fig. 4e). The multiple beams emitted with energetic electrons from the cathode have varying intensities corresponding to the irregular light emission from the bare GE surface areas. At the same time all low-energy electrons are focused onto the axis (Fig. 4e). Focusing of the total beam to a minimum spot size is achieved with a $-0.5 \mathrm{kV}$ electrostatic-lens potential and with a $+3 \mathrm{kV}$ post-accelerating potential (Fig. $4 \mathrm{f}$ ). In reverse-field mode a visible electron beam appears during the $\mathrm{P}_{\mathrm{s}}$-switching on the luminescent screen only when an extraction potential is applied. The optical inspection also proves that the insulation at the edges of the grid electrode efficiently suppresses the electron emission there.

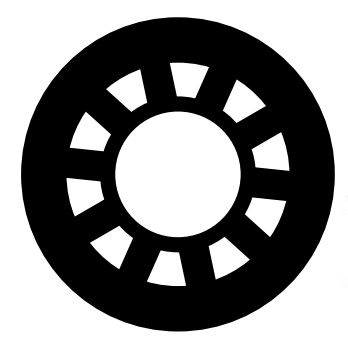

a)

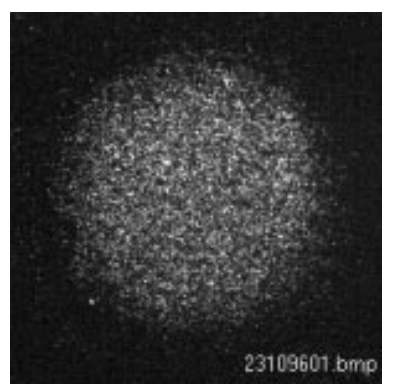

d)

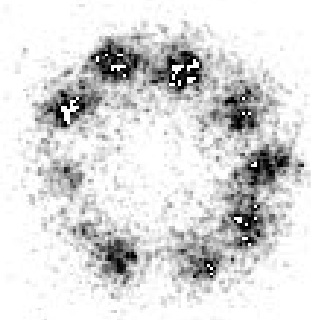

b)

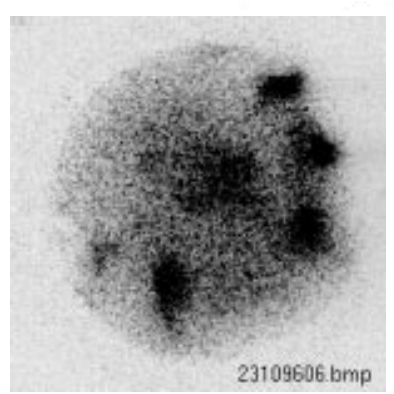

e)

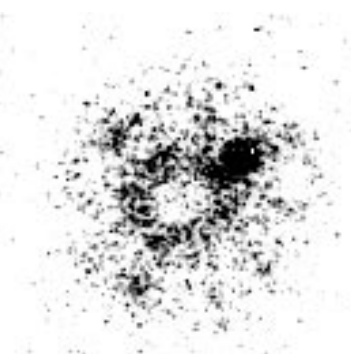

c)
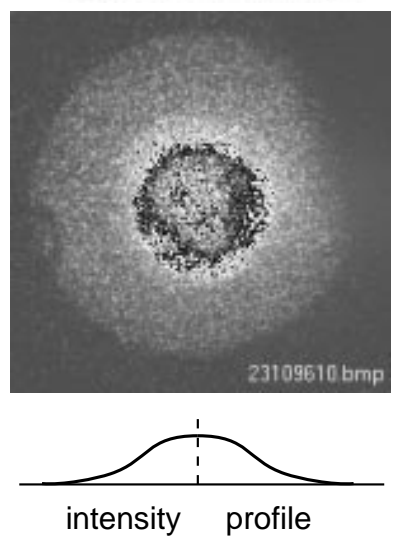

f)

FIG. 4. Fast photography of the GE: the shape of the grid electrode (a), with FE self-emission (b), and with reverse-field excitation (c). The emitted electron beam hitting a luminescent screen above the GE surface in selfemission without focusing is shown in (d), with focusing $(-2 \mathrm{kV})$ and post-acceleration $(+1 \mathrm{kV})$ in (e), and with minimum spot size focusing a potential of $-0.5 \mathrm{kV}$ and an acceleration potential of $+3 \mathrm{kV}$ in (f). 
For technical applications it is important that FE emission can be easily controlled in space and time, as demonstrated with the emission of a double pulse of $25 \mathrm{~A} / \mathrm{cm}^{2}$ amplitude spaced by $2 \mu \mathrm{s}$, as shown in Fig. 5 [13]. Since the rapid change of spontaneous polarization

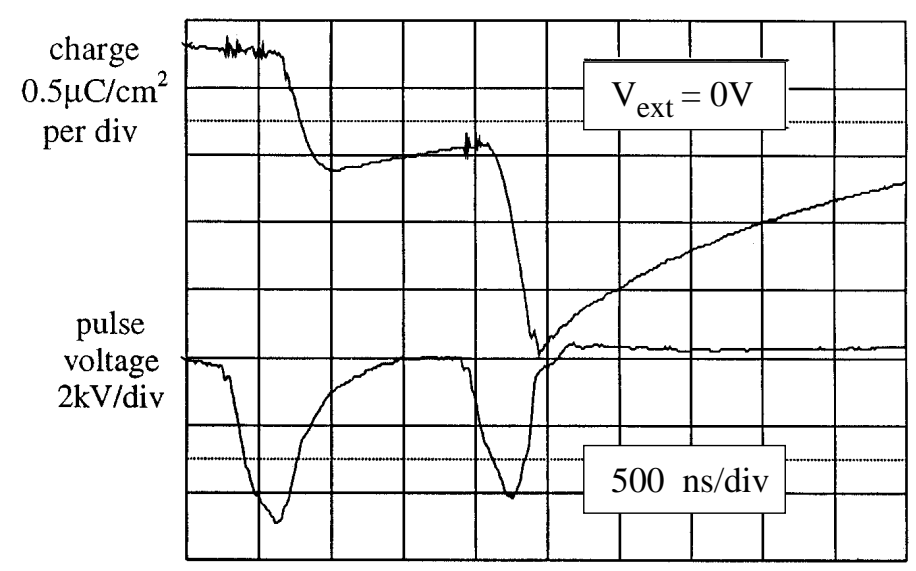

FIG. 5. FE self-emission with $0.6 \mathrm{MHz}$ repetition rate from a PLZT cathode without reset. Top: emitted charge density (with peaks of 0.9 and $1.5 \mu \mathrm{C} / \mathrm{cm}^{2}$ ). Bottom: $\mathrm{HV}$ pulse waveform ( $4.2 \mathrm{kV}$ amplitude).

$\Delta \mathrm{P}_{\mathrm{s}}$ is characterized by a hysteresis curve, the FE electron emission starts only above a defined switching threshold of thermal, mechanical, optical or electrical excitation energy. FE emission can only be usefully applied when the excitation threshold is lower than the destruction and breakdown limits of the FE material. The spatial distribution of switched FE domains determines also in a not fully FE sample the average intensity of electron emission. The amount of polarization $\Delta \mathrm{P}_{\mathrm{s}}$ switched in and averaged over the whole $\mathrm{FE}$ cathode volume can be approximately expressed by

$$
\Delta \mathrm{P}_{\mathrm{s}}=\left(\delta^{3} \mathrm{P}^{\prime \prime} / \bar{h} \mathrm{~F}_{\mathrm{s}}\right) \times \int_{0}^{F_{\mathrm{s}} \bar{h}} \int_{0}^{\prime} \mathrm{m}^{\prime} \mathrm{df} \mathrm{dx}
$$

where $\mathrm{P}^{\prime \prime}$ is the average polarization change and $\delta$ the size of a single $\mathrm{FE}$ domain, $\mathrm{F}_{\mathrm{s}}$ the total switched area and $\bar{h}$ the effective thickness of the cathode. The line number density $\mathrm{n}^{\prime}=\mathrm{dn} / \mathrm{dx}$ and the cross-sectional number density $\mathrm{m}^{\prime}=\mathrm{dm} / \mathrm{df}$ describe the distribution of switchable FE domains perpendicular and parallel to the emitting surface GE, respectively. The domain size of polycrystalline FE material is normally smaller or at most equal to the grain size. The average kinetic energy of the electrons emitted from a sample of effective thickness would be given according to Eq. (3) by the average potential $\Delta \bar{V}_{s}=\Delta \mathrm{P}_{\mathrm{s}} \times \bar{h} / \varepsilon$ generated on the cathode surface. However, sinc the individual domains in a polycrystalline FE material seem to switch almost fully their polarization $\mathrm{P}^{\prime \prime}=\mathrm{k} \mathrm{P}_{\mathrm{s}}$, with $\mathrm{k}<1$, much higher kinetic energies are observed even at weak average emission currents. Strong emission is only achieved when a large number of individual FE domains are simultaneously switched together, created or annihilated, within a submicrosecond time interval. In reality the FE emission is strongly dependent on the effective thickness $\bar{h} \leq \mathrm{d}$, the electrode type and shape, and the electric field distributions inside, outside and on the surface of the sample before and after switching. The strong electrically-induced emission from FE samples covered with metallic grid electrodes is very efficient, but weak FE emission has also been observed from a bare FE surface without any electrode or conducting material (for example, $[3,5,26]$ ). The latter type of electron emission cannot be induced with 
any of the alternative emission methods [14-22] using reverse field excitation, which rely on the field emission of electrons from the FE-metal interfaces of the GE grid electrode [27].

\subsection{Relaxing back of polarization and restoration of screening charges}

Repetitive switching of an FE sample with the same HV polarity as shown in Fig. 5 may regularly induce FE self-emission without applying any reset pulse with opposite polarity to the cathode. For such an operating mode the cathode may require special preparation by prepoling, which creates an internal space-charge field which draws back $\mathrm{P}_{\mathrm{s}}$ into the original direction. Hence, the initial switching of $\mathrm{P}_{\mathrm{s}}$ must be followed by the relaxing back of $\mathrm{P}_{\mathrm{s}}$ to the original state. The next emission pulse is then generated with the same amount of switched $\Delta \mathrm{P}_{\mathrm{s}}$ as the previous one. Polarization and relaxing phases can be clearly observed if the FE emission is induced near the switching threshold, as shown for two cathodes with different types of ceramic PLZT x/y/1-y material in Fig. 6. The numbers $x$ and $y$ refer to atom percentage of the constituents of the PLZT material, characterized by the notation: $\left(\mathrm{Pb}_{1-\mathrm{x}}, \mathrm{La}_{\mathrm{x}}\right)\left(\mathrm{Zr}_{\mathrm{y}}, \mathrm{Ti}_{1-\mathrm{y}}\right) \mathrm{O}_{3}$. Note that in previous publications [6-12] the FE ceramics PLZT 4/95/5 was presented with the notation PLZT 2/95/5, since the lanthanum content was given in mol $\%$ instead of atom $\%$.

Figure 6a shows the FE electron emission induced by switching and relaxing back of a PLZT 8/65/35 cathode, which is mainly ferroelectric at room temperature. The emission sequence shown in Fig. 6 b for a PLZT 4/95/5 sample is characterized by a double peak caused by $\mathrm{P}_{\mathrm{s}}$-switching during the leading edge of the exciting $\mathrm{HV}$ pulse (first peak) and by relaxing back of $\mathrm{P}_{\mathrm{s}}$ during the decay time of the $\mathrm{HV}$ pulse. Both events cause an emission pulse from the surface of the cathode. The second electron current pulse is the result of the combined action of the negatively charged GE electrode after relaxing of $P_{s}$ and of the negative tail of the HV pulse exerted by the cathode on the surface plasma which has formed on the GE during the first emitted pulse. After the decay of the HV pulse, polarization changes may continue, and even after several microseconds positive (as shown in Fig. 6a) or negative charges can still be expelled from the cathode surface. Such after-effects are particularly observed with PLZT $\mathrm{x} / 65 / 35(\mathrm{x}=7,8,9$ atom percentage) ceramics which are therefore less suitable for highrepetition-rate applications [8].

a)
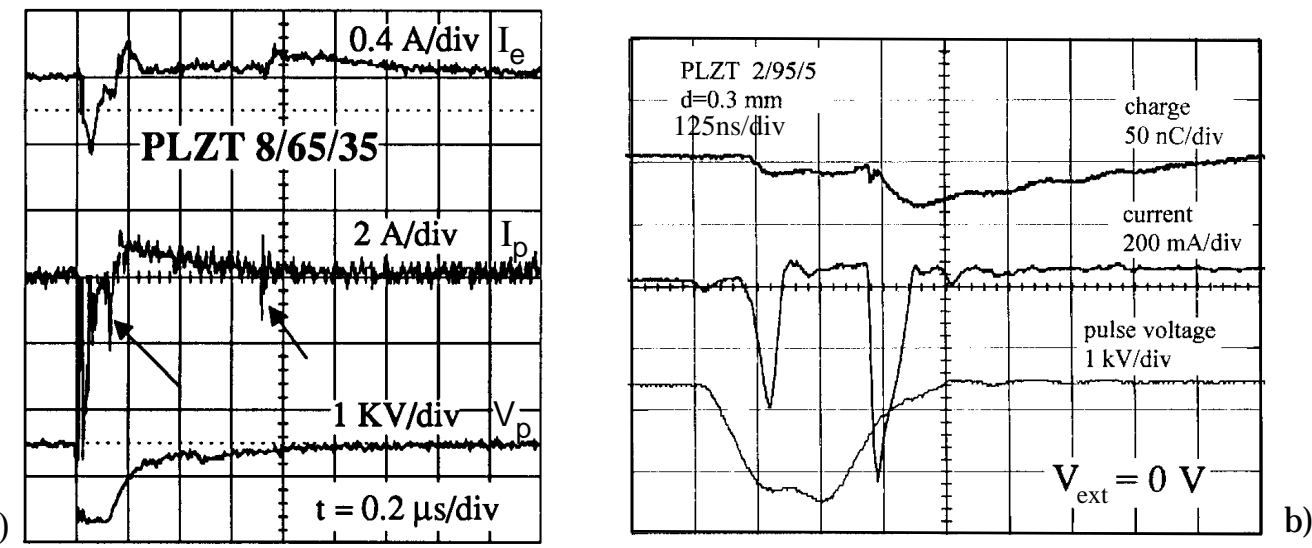

FIG. 6. (a) Charging (switching) current $\mathrm{I}_{\mathrm{p}}$ and emission current $\mathrm{I}_{\mathrm{e}}$ induced by $\mathrm{P}_{\mathrm{s}}$-switching and backward relaxing with $\mathrm{V}_{\text {ext }}=0$ from a PLZT 8/65/35 cathode [28], (top: emitted current $\mathrm{I}_{e}$, middle: charging current $\mathrm{I}_{\mathrm{p}}$, bottom: HV pulse, $0.2 \mu \mathrm{s} /$ div); (b) from a PLZT 4/95/5 cathode (top: emitted charge $\mathrm{Q}_{\mathrm{e}}$, middle: $\mathrm{I}_{\mathrm{e}}$, bottom: HV pulse, $0.125 \mu \mathrm{s} / \mathrm{div})$. 
PLZT 4/95/5 contains FE domains dispersed in an essentially antiferroelectric (AFE) matrix at room temperature. However, under the influence of a pulsed electric field above 20 to $25 \mathrm{kV} / \mathrm{cm}$, fast phase transition of AFE domains into the FE state sarts even at room temperature on a nanosecond time-scale. The FE phase formation is also rapidly reversible (Fig. 6b) through FE domain annihilation when the HV pulse amplitude has dropped below the $\mathrm{P}_{\mathrm{s}}$-switching threshold. It has been shown that forward and backward switching by electricfield-induced phase transition has the same effect as direct switching and relaxing back of $\mathrm{P}_{\mathrm{s}}$ with HV pulses [28]. In zirconium-rich PLZT ceramics, FE microdomains are induced and annihilated at a subnanosecond time-scale within a rather large temperature range below and even above the ferroelectric-paraelectric phase transition temperature [29].

At high HV-pulse amplitudes with PLZT 4/95/5 no further emission pulses are observed after the HV pulse has decayed as the induced FE domains disappear if the excitation field goes to zero. With PLZT 8/65/35, on the other hand, the local interaction of active FE domains and free charges from the surface plasma may continue far beyond the duration of the HV switching pulse. For both materials the two separate emission peaks merge into a single strong emitted electron pulse if the excitation field is significantly raised above the threshold level (Fig. 2). The phases of $\mathrm{P}_{\mathrm{s}}$-switching and relaxing back are then overlapping in time, but not necessarily in the same locations of the GE. Any rapid change of $P_{s}$ is equivalent to the appearance of strong electric surface charges and fields, which not only start off electron emission, but also initiate charge compensation processes which, after the charge losses induced by the emission, tend to remove any local non-equilibrium of charge distribution and of the cathode as a whole. Possible ways of charge compensation are by the formation of a compensated polydomain structure in the FE bulk, by internal mobile charge carriers such as electrons, holes and ions, and by external electrons getting attached to the surface from the electrodes or from the surface plasma. The way in which the surface is recharged with new charges to compensate for the polarization fields and the charge loss after an emission pulse strongly influences the next emission pulse. For high intensity, high repetition rates and good reproducibility of the FE emission, recharging with electrons from the surface plasma or from the edges of the GE electrodes is most efficient. Some authors call these compensating charges 'electrons in surface states'. Many experiments (for example Refs. [7, 12, 30]) have revealed the major importance of externally-provided compensating electrons for a well-functioning, intense FE emission under any conditions of excitation. Compensating 'surface' electrons are not only more easily emitted, but also withdrawn more easily via the sample electrodes by the excitation voltage which induces $\mathrm{P}_{\mathrm{s}}$-switching. Compensation with bulk-internal electrons or defect centres (e.g. oxygen vacancies) partially immobilizes the otherwise switchable domains, thus raising the threshold of the switching voltage. Another internal compensation process is, for example, the mutual screening between adjacent FE domains (e.g. twin domains). External excitation and extraction fields, fields due to $\mathrm{P}_{\mathrm{s}}$-switching and charge compensation processes strongly interact during and after each emission event until the average $\mathrm{P}_{\mathrm{s}}$ has relaxed back and overall charge neutrality has been reached again. Unpoled cathodes often require a reset pulse to restore the original polarization state, although after repeated unipolar HV-pulse excitation the average resultant $\mathrm{P}_{\mathrm{s}}$ may be directed in a way similar to that after a prepoling treatment. In reverse-field emission mode the interaction of Pswitching, charge compensation and extraction fields leads to more complicated patterns of electron emission and screening (Fig. 3). 


\subsection{FE emission and surface plasma}

FE emission mechanisms provoke the formation of surface plasma as is the case for any other type of intense electron emission. If the local emission strength becomes very high when FE domains are switching this always seems to be the case - then surface material including adsorbed gas molecules is sputtered and released from the emitting surface and also from the electrodes hit by energetic electrons accelerated in the local surface fields. Before switching a fine-grained polycrystalline FE sample, the field configuration on the surface is mainly determined by the applied HV pulse and the dielectric permittivity of the sample. Qualitative computations (for example, in Ref. [31]) show that the averaged field configuration on the surface of a high- $\varepsilon$ material is characterized by a strong enhancement near the electrode edges and by a bend of the surface field away from the edge into the direction parallel to the emitting surface. After $\mathrm{P}_{\mathrm{s}}$-switching the local and average field distribution changes drastically and the uncompensated polarization fields become the determining factor. The field strength near the electrode edge will be higher by orders of magnitude, compared to areas far away from the edge where no switching has occurred yet. The strong fields not only accelerate the electrons from the bare surface towards the electrode edges, but the sputtered ions are also accelerated along the surface in the opposite direction, i.e. away from the electrode edge. The ions form a fast-moving, virtual space-charge anode above the bare FE surface (Fig. 7) like the

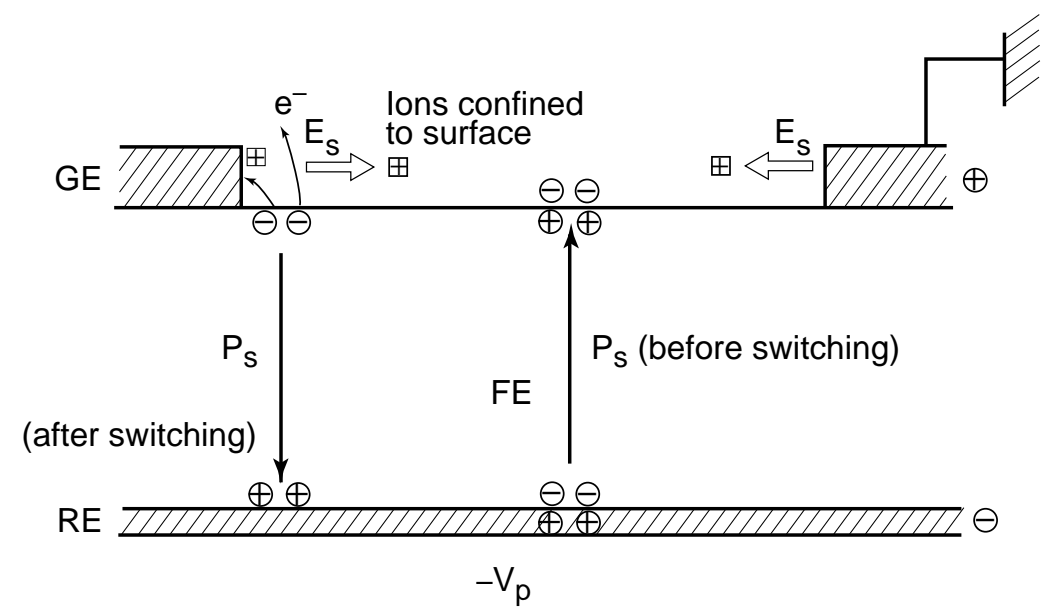

FIG. 7. Confined surface plasma formed by the polarization fields and the charged particle dynamics after FE switching has started in self-emission mode with negative excitation voltage $-V_{p}$ to $R E$.

metal electrodes on top of the GE surface. One can consider this special type of plasma as being formed by counter-moving electron and ion beams. The plasma tends to spread parallel to the cathode surface, whilst acting as a positive electrode which is transparent for the electrons emitted and enhancing FE switching and emission from the area covered during its expansion as optically observed and shown in Fig. 4b. After switching new bare FE surface areas the polarization field holds back the plasma ions from escaping into the vacuum gap with the expelled electrons. The initial field configuration, which was valid at the metal edge, continues to propagate like a wave until the whole free FE area is switched. Figures $4 \mathrm{~b}$ and $4 \mathrm{e}$ show the light and electron emission in FE self-emission mode occurring from the bare FE surface areas. The metallized and insulated regions of the GE remain dark and do not emit electrons. Similar observations are reported in Ref. [32] and the effects have been proposed for application in flatpanel displays [33]. During the whole expansion process the plasma forms a nearly 
homogeneous thin sheet confined to the bare FE surface and partially screens the negative surface charge, provided very strong switching and extraction fields are avoided. When the switched polarization of the FE sample starts to relax back, the plasma favours charge compensation and serves as a reservoir for restoring via the grid electrodes the charges 'lost' by electron emission.

Figure 8 shows that different processes occur when the FE cathode is poled in the opposite direction (cf. Fig. 7) and when it is pulsed with reverse-field direction (positive HV pulse to the rear electrode RE [21]). The reversed field switching generates a polarization field pattern which is characterized by identical strength, but by a reversed direction compared to the excitation of FE emission with negative HV pulses to RE. We observe (Fig. 4c) that electrons are now emitted from the metal edges of the grid electrode towards the oppositely-charged FE surface areas at a threshold field which is lower than for FE self-emission, apparently owing to the field enhancement caused by the thin metal electrodes. Ions are sputtered from the free FE surface and from rest gas adsorbed at the surface and accelerated towards the metal edge where new electrons are sputtered off. At the same time the ions are pushed away from the FE surface by the strong polarization field, hence the plasma has a tendency to expand into the vacuum gap.

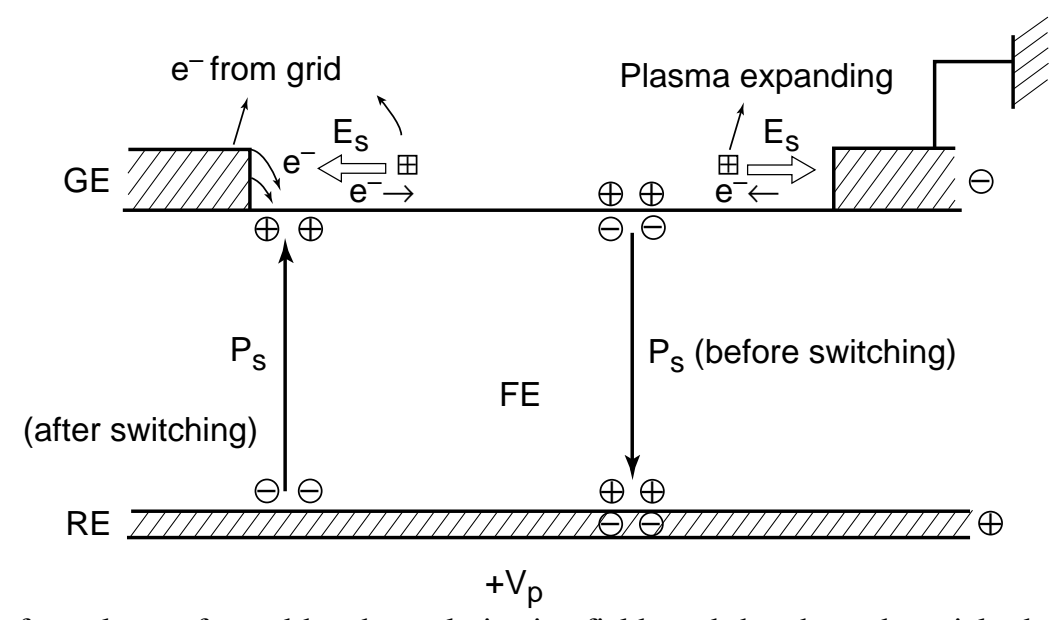

FIG. 8. Expanding surface plasma formed by the polarization fields and the charged particle dynamics after FE switching has started in reverse-field mode with positive excitation voltage $+\mathrm{V}_{\mathrm{p}}$ to $\mathrm{RE}$.

Also in the reverse-field mode the switching may proceed across the GE surface, while electrons form the moving space-charge wave. However, no self-emission is observed, since there is no field component driving the electrons away from the surface as in the case of FE emission. With an external potential, electron current may be extracted from the plasma, but simultaneously the plasma expansion is enhanced and vacuum-gap breakdown is favoured.

When $\mathrm{P}_{\mathrm{s}}$ starts to relax back under reverse-field conditions, electron emission induced by the negative potential appearing on the FE surface can be observed. Such a case, which resembles real FE emission, is shown in Fig. 9. The surface plasma screens the polarization fields so effectively that electrons are expelled through and from the plasma with high intensity, but with much less kinetic energy than under FE emission conditions. Metallic elements on the GE surface emit as well and the electron current emission is induced by the negative surface potential appearing after relaxing back of $\mathrm{P}_{\mathrm{s}}$. 


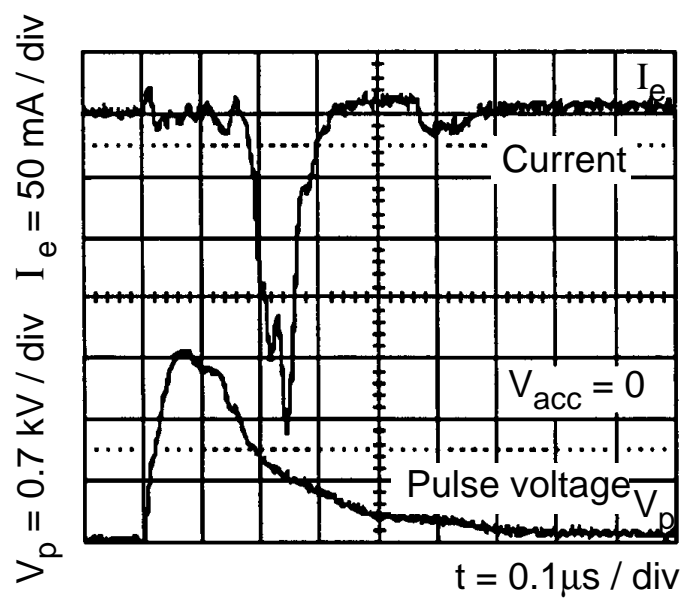

FIG. 9. Electron emission from a negatively-charged FE surface during relaxing back of polarization after reversed-field excitation of a PLZT 8/65/35 cathode [21].

Electron emission without $\mathrm{P}_{\mathrm{s}}$-switching and independent of polarity can be also achieved by the extraction of electrons from the arc plasma, which is formed if the conditions for vacuum or gas breakdown between the edges of the metal electrodes and the FE surface are reached before $\mathrm{P}_{\mathrm{s}}$-switching starts [22]. By measuring the charging and switching currents of the FE cathode and by optical inspection of the cathode surface one can experimentally determine which of the alternative excitation processes occurs first and the sequence of processes in any of the emission modes. The current density jemitted from a cathode covered by plasma is determined by Bohm's equation

$$
j=\operatorname{aen}_{e} \sqrt{\frac{k T_{e}}{m_{i}}}
$$

with $\mathrm{a} \approx 1, \mathrm{~T}_{\mathrm{e}}$ is electron temperature and $\mathrm{m}_{\mathrm{i}}$ is ion mass. Equation (4) may explain the two orders of magnitude increase of emitted current density compared with the Langmuir-Child current density. Such an enhancement of emitted current density can be observed, if an extraction field is applied and if the plasma density $\mathrm{n}_{\mathrm{e}}$ is sufficiently high (as for example reported in [15], [16], [18], [19]). The enhancement effect of the surface plasma can also be recognized in FE self-emission mode (for example in Fig. 5) where the amplitude of the second pulse is greater than that of the first owing to the previously generated surface plasma, and in spite of a smaller excitation voltage amplitude.

After surface plasma formation care must be taken, even in pure FE emission mode, not to load the sample with too much excitation energy in excess of the necessary energy for polarization switching; otherwise the risk of destroying the grid electrodes and the FE sample surface is high. Similarly, strong d.c. or low-frequency external (extraction) fields should not be used together with strong FE emission, as plasma formation will be strongly enhanced and its expansion into the direction of the emitted electron beam will cause breakdown in the beam transport structure. The use of strong HV pulses with positive or negative polarity to the grid electrode on the emitting cathode surface GE is not recommended, since this mode has proven to be destructive for the cathode in several applications. 


\subsection{Laser-induced FE emission}

Two laser-induced electron emission modes have been investigated: 'normal' laserinduced emission with repetitive illumination of an FE photocathode under a d.c. extraction field, and an intense 'self-emission' mode without externally applied extraction field, shown in Fig. 10a [31], which was predicted in [34]. Intense electron emission can be observed following a $\mathrm{P}_{\mathrm{s}}$-switching excited by an HV pulse without d.c. extraction potential $[10,12,30$, $31,35]$. Figure $10 \mathrm{~b}$ shows the electron current emitted from a PLZT 2/92/8 sample illuminated

a)
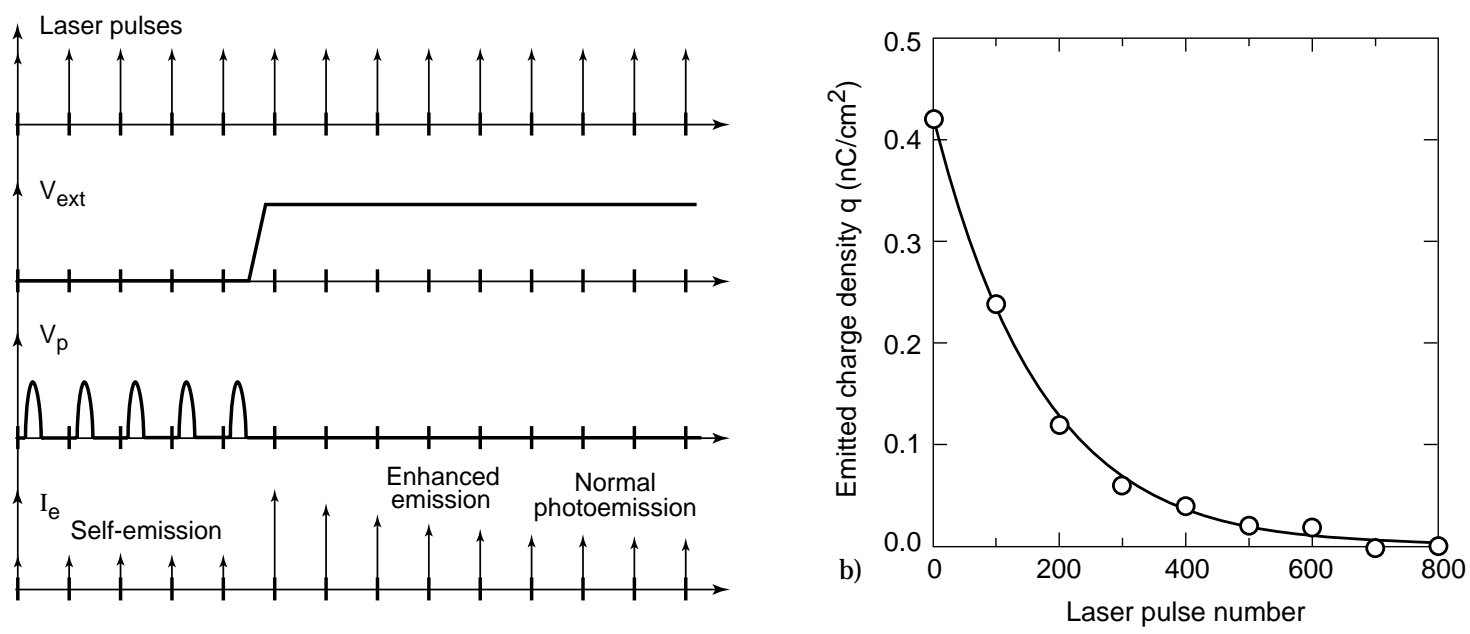

FIG. 10. a) Principle of laser-induced electron self-emission [31]. b) Self-emitted charge density q from a PLZT 2/92/8 FE photocathode decaying with the number $n_{L}$ of 140 fs pulses at $780 \mathrm{~nm}$ wavelength from a Ti-sapphire laser under zero extraction field [12]. The decay is exponential: $\mathrm{q}_{\mathrm{e}}=\mathrm{q}_{0}$ exp $\left(-\mathrm{kn}_{\mathrm{L}}\right)$ with $\mathrm{k}=0.0055$.

with $140 \mathrm{ps}$ laser-light pulses of a Ti:sapphire laser at $780 \mathrm{~nm}$ wavelength in the absence of an extraction field [12], but after previous electric-field-excited $\mathrm{P}_{\mathrm{s}}$-switching. In intense laserinduced self-emission, electrons can be ejected with kinetic energies of several $\mathrm{keV}$ and with current densities of several $\mathrm{A} / \mathrm{cm}^{2}$. As in field-excited FE emission a threshold of laser intensity is observed, below which laser-induced 'self-emission' cannot be detected (Fig. 11).

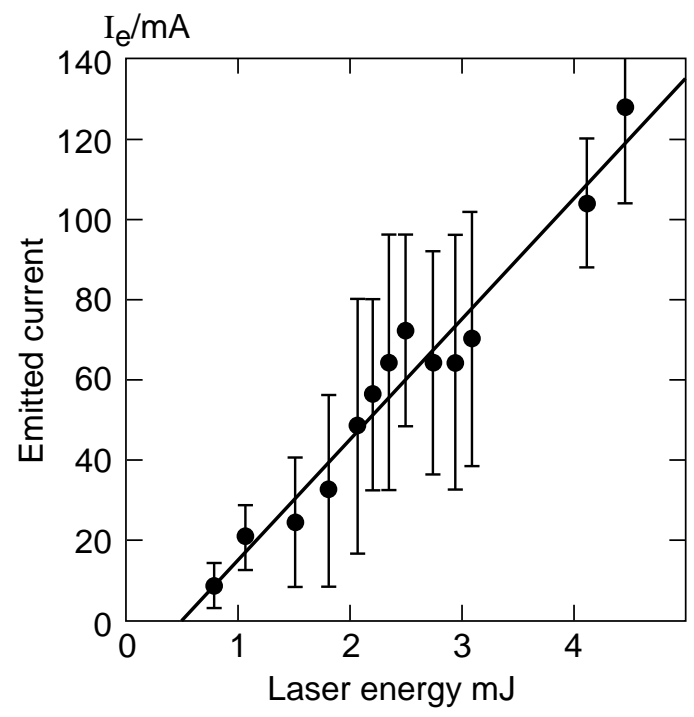

FIG. 11. Threshold and dependence of self-emitted electron-beam current on laser pulse energy at $355 \mathrm{~nm}$ from a PLZT 4/94.5/5.5 cathode with interleaved, electric-field-excited $\mathrm{P}_{\mathrm{s}}$-switching. 
The appearance of highly energetic electrons without the application of an extraction potential, and the observation of a threshold for laser-induced FE emission are clear signatures of $\mathrm{P}_{\mathrm{s}}$-switching caused by the light intensity of the laser pulse. The emitted electrons with kinetic energy of the order of $\mathrm{keV}$ must have necessarily passed through a potential difference $\Delta \mathrm{V}_{\mathrm{s}}$ (Eq. 1) equivalent to their final kinetic energy. The depth a, towards which the fast $\mathrm{P}_{\mathrm{s}}-$ switching progresses inside the bulk of the FE cathode, depends on the absorption length of the material for the applied laser frequency. It is generally much smaller than the sample thickness $d$ for UV light, unlike for longer wavelengths which are only weakly absorbed by the material. Reference [10] reports also a different type of laser-induced FE emission with $\mathrm{P}_{\mathrm{s}}$-switching of the whole bulk of a 0.5 -mm-thick fully FE cathode. This particular emission event lasts for several tens of nanoseconds and seems to be caused by a polarization wave proceeding with the sound velocity of $3.5 \mathrm{~km} / \mathrm{s}$ through the FE sample. The fast switching which appears in laserinduced self-emission seems, on the other hand, to be determined by the penetration velocity $\mathrm{c} / \sqrt{\varepsilon_{\mathrm{r}}}(\approx 50 \mu \mathrm{m} / \mathrm{ps})$ of the electromagneticwave sweeping through the matrix of a sample with dispersed FE domains. In this case $\mathrm{P}_{\mathrm{s}}$-switching is induced by the wavefront wherever it encounters FE domains, which are individually switched with sound velocity during a time interval given by the domain dimensions.

If an additional extraction potential is applied, the self-emission intensity may be enhanced by more than two orders of magnitude [30]. The self-emission and the enhancement effects can only be observed after a previous HV-pulse-excited $\mathrm{P}_{\mathrm{s}}$-switching of the FE cathode, and the laser-induced self-emission disappears after a few or many hundreds of laser shots if the cathode is not regularly refreshed by interleaved HV-pulse switching. This behaviour is the result of the charge compensation processes following the excitation pulse either by electric field or by laser. The HV-pulse excitation leads to a very violent $\mathrm{P}_{\mathrm{s}}$-switching. The electrons which are attracted from the grid electrode and from the surface plasma compensate for the apparent polarization field on the bare FE surface by entering surface states. These electrons can be more easily emitted under subsequent laser irradiation than electrons from the bulk. Repeated laser illumination under d.c. field, on the otherhand, causes compensation with bulk-internal mobile charge carriers which are much more difficult to extract or to expel. The charge density emitted in laser-induced FE self-emission mode $\left(2 \mathrm{~A} / \mathrm{cm}^{2}\right)$ reported in Refs. [10, 12, 35] has not yet reached the maximum charge densities obtained with HV-pulse-excited emission (> 100 $\mathrm{A} / \mathrm{cm}^{2}$ ). Several possible reasons may be identified: (a) the number density of the domains which undergo $\mathrm{P}_{\mathrm{s}}$-switching or fast phase transitions is smaller in the case of optical switching than under field-excited excitation; (b) the laser-induced $\mathrm{P}_{\mathrm{s}}$-change only occurs in a finite layer smaller than the thickness $d$ of the bulk of the cathode, and c) strong HV-switching mainly leads to surface charge compensation with electrons in surface states, whereas laser-induced self-emission includes compensation processes by internal, mobile charges. It has been observed that laser illumination of FE cathodes under a d.c. extraction potential strongly degrades the $\mathrm{P}_{\mathrm{s}}$-switching capability of an FE cathode in the region of the laser spot $[12,35]$ and has even reproducibly reversed the poling direction in this area.

Since laser-induced FE self-emission closely resembles HV-excited FE emission it is obvious that similar electrodynamic conditions are valid during the emission phase. In both modes of excitation the 'surface state' electrons compensating for the FE polarization have to be removed before inducing $\mathrm{P}_{\mathrm{s}}$-switching. This is achieved via the grid electrodes on the GE in 
field-excited emission. Figure 12 shows that the efficiency of normal (without $\mathrm{P}_{\mathrm{s}}$-switching) laser-induced emission from FE cathodes rises steeply with laser power density for all wavelengths. Hence, under the impact of the laser pulse the compensating electrons are virtually 'evaporated' by (high-intensity) photoeffect and then heated up by the strong laser intensity $\mathrm{W}_{\mathrm{L}}$ corresponding to an electric laser field $\mathrm{E}_{\mathrm{L}}$ :

$$
\mathrm{E}_{\mathrm{L}}[\mathrm{V} / \mathrm{cm}]=\sqrt{1 /\left(\mathrm{c} \varepsilon_{0}\right)} \times \sqrt{\mathrm{W}_{\mathrm{L}}}=18.9 \times \sqrt{\mathrm{W}_{\mathrm{L}}\left[\mathrm{Watt} / \mathrm{cm}^{2}\right]} .
$$

At a medium-range laser power flux of $1 \mathrm{GW} / \mathrm{cm}^{2}$, a field amplitude of $10^{7} \mathrm{~V} / \mathrm{m}$ is generated in the laser spot, sufficient to initiate electron 'evaporation' and $\mathrm{P}_{\mathrm{s}}$-switching.

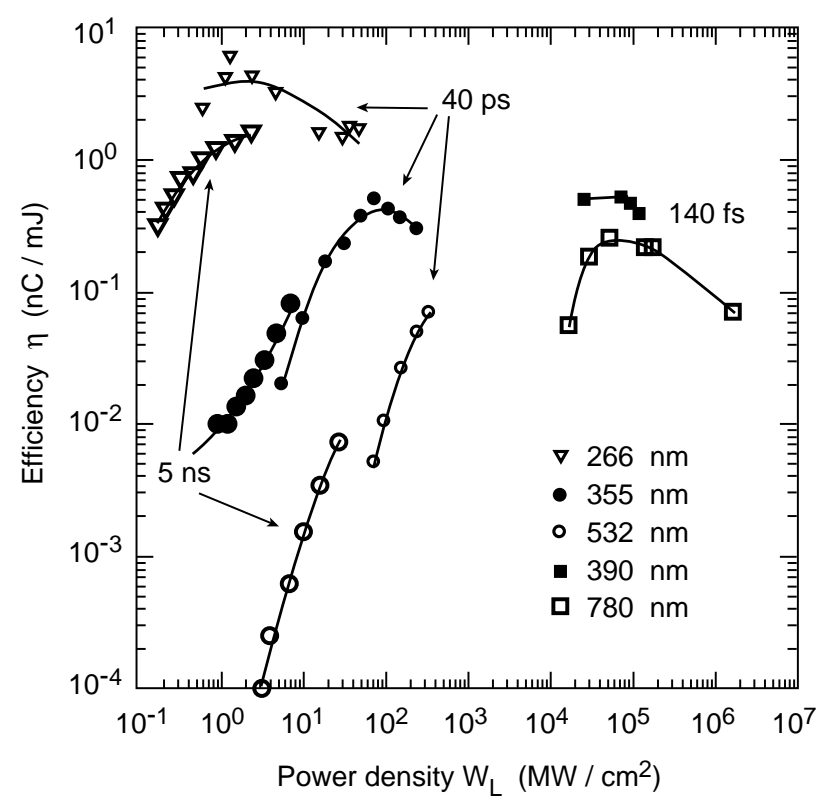

FIG. 12. Emission efficiency of normal photoemission from a PZT $95 / 5$ cathode doped with $2 \% \mathrm{Cr}_{2} \mathrm{O}_{3}$ for different wavelengths and laser pulse lengths as a function of laser power density at $30 \mathrm{kV}$ extraction voltage.

The resulting electron cloud is displaced from the GE surface leaving a positively charged surface behind, as appears in field-excited emission after removal of the compensating electrons by the positive potential applied to grid electrode GE. In both modes a positive surface potential drives the initial change of $\Delta \mathrm{P}_{\mathrm{s}}$. Observations with classical photoemission show no delay or difference in waveform between photoelectron current and laser light intensity signals. Down to laser pulse rise times of $0.1 \mathrm{~ns}$ good congruence of the rise times of the emitted current pulse and of the laser intensity waveforms has been observed with the laser-induced FE self-emission mode [35]. The light velocity $\mathrm{c} / \sqrt{\varepsilon_{\mathrm{r}}}$ seems to be the main parameter determining the relative timing of laser-induced excitation on the one hand and of the FE switching and the resultant electron emission on the other hand. Laser-induced FE self-emission has been also initiated with 140 fs pulses with wavelengths in the visible and the infrared range [11], but it has not been verified whether there is a time lag or a distortion of the current signal with respect to the laser intensity waveform at this ultra-short time-scale. The fall time of an intense self-emitted electron beam pulse is generally distorted because of space-charge forces in the vacuum gap [9, $10,31]$, even at nanosecond laser pulse lengths of $1 \mathrm{~A} / \mathrm{cm}^{2}$ emitted-electron current density.

The observed decay of the self-emission beam current amplitude with the number of laser pulses under zero extraction voltage and without surface charge refreshment by interleaved $\mathrm{P}_{\mathrm{s}}$ - 
switching may be explained by the gradual exhaustion of compensating 'surface state' electrons and their replacement by 'bulk-internal' charges. In the nanosecond range self-emission went to zero after as few as 3 to 5 laser pulses [30]. The shorter the laser pulse the more electron pulses can be generated in self-emission mode before the available electrons are exhausted. At the highest power level in the femtosecond range weak self-emission was observed even without interleaved $\mathrm{P}_{\mathrm{s}}$-switching.

The term quantum efficiency (QE) can only be used artificially in strong laser-induced FE self-emission, e.g. for comparing FE with conventional photocathodes. Physically, QE is rather meaningless in FE self-emission, since the emission intensity is characterized by a threshold and, hence, is not proportional to the number of impinging photons, as is the case in the classical photoeffect. Laser-induced FE self-emission functions by $\mathrm{P}_{\mathrm{s}}$-switching caused by the incident electromagnetic radiation of any wavelength, provided a significant part of the incident energy is absorbed inside the FE photocathode material and the threshold energy for switching is exceeded. Therefore we define the efficiency for laser-induced FE emission more conveniently as $\eta=$ (emitted charge/nC)/(laser-beam energy/mJ) and keep this definition also for 'normal' laser-induced FE emission under d.c. extraction field. Nevertheless QE can be artificially calculated from $\eta$ with Planck's constant $h$ and the laser frequency $v$ by $\mathrm{QE}=\eta \mathrm{e} /(h \mathrm{v})$.

The characteristics of 'normal' laser-induced emission with d.c. extraction field have been reported in Refs. [7, 8, 10, 28, 30]. Normal laser-induced FE emission resembles classical photoemission; hence, no apparent $\mathrm{P}_{\mathrm{s}}$-switching is visible. As in laser-induced FE selfemission mode and as in classical photoemission from metallic cathodes, the leading edge of the waveforms of the electron-beam current signal produced with a cathode in normal FE emission mode and the exciting laser pulse intensity waveform have the same shape. In experiments with different laser pulse lengths it was shown that the emission efficiency $\eta$ for this mode increases with laser power density by several orders of magnitude. This holds especially for longer wavelengths in the visible and the infrared range. Figure 12 (from Ref. [10]) shows the emission efficiency at $30 \mathrm{kV}$ extraction voltage of one particular FE photocathode as a function of laser power density obtained with 5-ns, 40-ps and 140-fs long laser pulses at 266, 355, 390, 532 and $780 \mathrm{~nm}$ wavelengths and with laser spot diameters on the FE target ranging from 0.5 to $5 \mathrm{~mm}$. At higher laser power density levels a flattening and subsequent decay of the efficiency $\eta$ is observed at all wavelengths independent of the laser system used. These limiting characteristics appear for shorter wavelengths at a lower power-density level. They are attributed to the space charge of the emitted electron beam at the given extraction voltage of $30 \mathrm{kV}$ applied in these experiments $[10,28]$. The maximum efficiency $\eta$ obtained under spacecharge limitation can be expressed by

$$
\log \eta=\log \left(\mathrm{k} \times \mathrm{V}_{\mathrm{ext}}\right)-\log \mathrm{t}_{\mathrm{p}}-\log \mathrm{W}_{\mathrm{L}},
$$

where $t_{p}$ is the laser pulse length and $\left(k \times V_{e x t}\right)$ is a function of the extraction potential. The power density also determines the switching and destruction thresholds, which, as well as the limit of the permanent plasma generation, grow with increasing power density of the excitation pulse. A large part of the results $[7,8,10,28,30]$ covers normal emission experiments under variable laser pulse conditions with FE photocathodes used in the classical way: laser illumination under a d.c. extraction field and with classical space-charge limitation. This scheme 
of employing FE photocathodes is the least interesting one, since the laser spot area on the cathode surface undergoes an unfavourable transformation into a poling state, which may completely inhibit $\mathrm{P}_{\mathrm{s}}$-switching. The 're-poling' effect which has been reproducibly observed under normal laser-induced FE emission conditions apparently contributes to the decay of emitted current density under self-emission conditions after a field-excited polarization switching. The correctly combined use of prepoling, polarization switching and self-emission schemes opens up the possibility of operating FE photocathodes at infrared wavelengths and at ultra-short laser pulse lengths with high efficiency and unequalled reliability.

\section{TECHNOLOGICAL FEATURES AND PROBLEMS OF FERROELECTRIC CATHODES}

The basic scheme of FE electron emission appears simple and strong emission has been observed with FE cathodes made of many different materials with strongly differing phase sequences and featuring a wide range of geometrical dimensions, electrode and surface characteristics and environmental conditions. However, the success or failure of achieving strong FE emission as defined in this article and dedicated to a particular technical purpose depends on the correct choice of a number of key parameters and environmental factors. For economic reasons only a few of them could be examined in more detail during the development work at CERN and these are dealt with below. The design and the preparation procedures of FE cathodes have to be optimized differently, whether high beam charge, strong emitted current and current density amplitudes, high beam quality (brightness and emittance), high precision in space and time, high repetition rates or long lifetimes are envisaged. The main requirements for FE cathodes in a typical application are:

- the precise control of the emission pulse intensity in space and time, for example, the optimization of maximum beam current amplitude for a given application;

- the control of the pulse repetition rate and synchronization with the external events;

- reliability and reproducibility, for robustness and strong fatigue resistance under severe environmental conditions and over a large temperature range;

- control of electron beam quality: homogeneity, brightness, emittance, energy spread and focusing properties of the electron beam pulses.

Control is carried out by the design and preparation procedures of the cathode, including the well-matched choice of the FE material and the correct choice of the mechanical, electrical and environmental parameters which interface the cathode to the system in which it has to be operated.

\subsection{FE material and cathode dimensions}

Though for basic studies of FE electron emission many different types of FE material, including organic single crystals such as TGS, have been investigated in the experiments at CERN, the cathodes for applications mainly consisted of PLZT polycrystalline ceramics. For optical applications (e.g. laser-driven photocathodes) PLZT x/65/35 (x = 7, 8, 9, 10) and PZT $5 / 95$ doped with a few percent of $\mathrm{Cr}_{2} \mathrm{O}_{3}$ have been frequently used [9, 10, 12, 30, 35], whereas the most widely chosen material for HV-pulse-excited emission (but also for laser-induced emission) was PLZT 4/95/5, which, at room temperature, is normally antiferroelectric and FE domains form only under electrical or mechanical stress. The phase transitions under $\mathrm{HV}$ 
excitation are so fast in this type of ceramics that very high pulse repetition rates have been achieved [7, 36]. The PLZT $x / 65 / 35$ class is less well suited for high repetition rate applications. In all perovskite FE materials the density distribution and the screening of switchable FE domains governing the P-switching threshold are very sensitive to heat treatments during the material production and sample preparation. This applies, for example, to the heating during sintering and other heating or cooling cycles in the course of surface electroding and insulating processes where screen-printing techniques are used. Also important for the $\mathrm{P}_{\mathrm{s}}$-switching and the excitation threshold are the grain size and the related domain size of the FE material. In the heat treatments during production and preparation of the material the size and distribution of FE domains can be controlled within wide limits from the subnanometre to the submillimetre range. A grain size between 1 and $10 \mu \mathrm{m}$ has proven to be a reasonable choice for the ceramic PLZT cathodes used for FE emission applications at CERN. In Refs. [12-19] PZT material with a Zr/Ti composition around 52/48, produced industrially for piezoelectric applications, has been used often for emission experiments with reverse field direction. Industrially available materials of this type are mostly doped with a few percent of $\mathrm{Nb}$ or Nd. Nevertheless, these FE ceramics can emit electrons in real FE mode above a high threshold. However, the elevated piezo-electric coefficients lead to strong mechanical vibrations and to fatigue after repeated $\mathrm{P}_{\mathrm{s}}$-switching cycles. Hence, cathodes made of this class of material were therefore more easily driven at lower excitation levels in the reverse-field emission mode.

Defects in the crystalline FE matrix generally have a negative influence on the $\mathrm{P}_{\mathrm{s}}$ switching. The freezing-in of high oxygen-vacancy concentrations by fast cooling down from a high temperature raises switching and emission thresholds so much that the HV-pulse excitation of electron emission becomes difficult or impossible at acceptable amplitudes. Oxygen vacancies and other crystalline defects and impurities can act as internal space-charge centres which may interfere with the charge compensation processes before and after a strong $\mathrm{P}_{\mathrm{s}}$ switching. Therefore, high-purity oxides have always been used for the production of the PLZT material, which was prepared for FE cathodes used at CERN.

In the past the choice of the FE cathode sample geometry (round ceramic discs of 16 to 20 mm diameter and 0.3 to $1 \mathrm{~mm}$ thickness) was successful for strong FE emission, although a careful optimization together with the electrode geometry was never followed through. Material homogeneity, low porosity and absence of mechanical defects, such as cracks and large holes, are necessary conditions for obtaining reliable and reproducible strong FE emission.

The outstanding switching and emission properties of the PLZT material class with an atom-percentage composition of 4/95/5 and characterized by a fine-grained though rather porous microstructure (Fig. 13a) with a distribution of FE domains at room temperature inside a mainly non-polar matrix, led directly to the attempt of using fine-grained (several $\mu \mathrm{m}$ ) $\mathrm{FE}$ powder material in a dielectric binding matrix for producing large area cathode layers. In Ref. [37] cathodes are described, which consist of a mixture of FE powder (e.g. $\mathrm{PbTiO}_{3}$ ) and plexiglass dissolved in chloroform prior to mixing as the non-polar binding material. These cathodes electroded by evaporated Au-grids have been operated successfully with electrical HVpulse excitation. Powder-type FE cathode layers, like cathodes made from partially FE materials (such as PLZT 4/95/5), have the practical advantages of low volume change and low fatigue during $\mathrm{P}_{\mathrm{s}}$-switching, and very fast response times to the excitation fields (see Section II.E). 
$5.0 \mu \mathrm{m}$

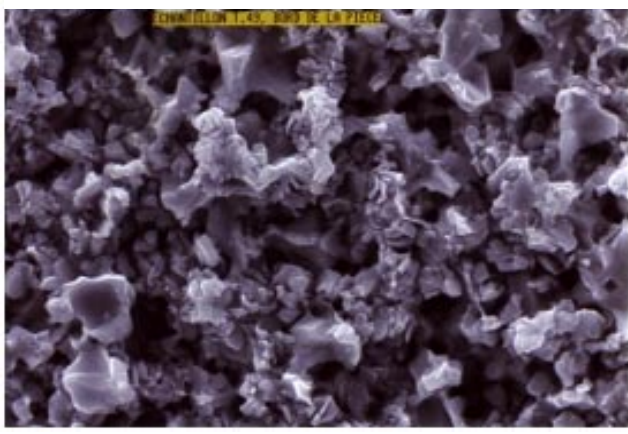

$10.0 \mu \mathrm{m}$
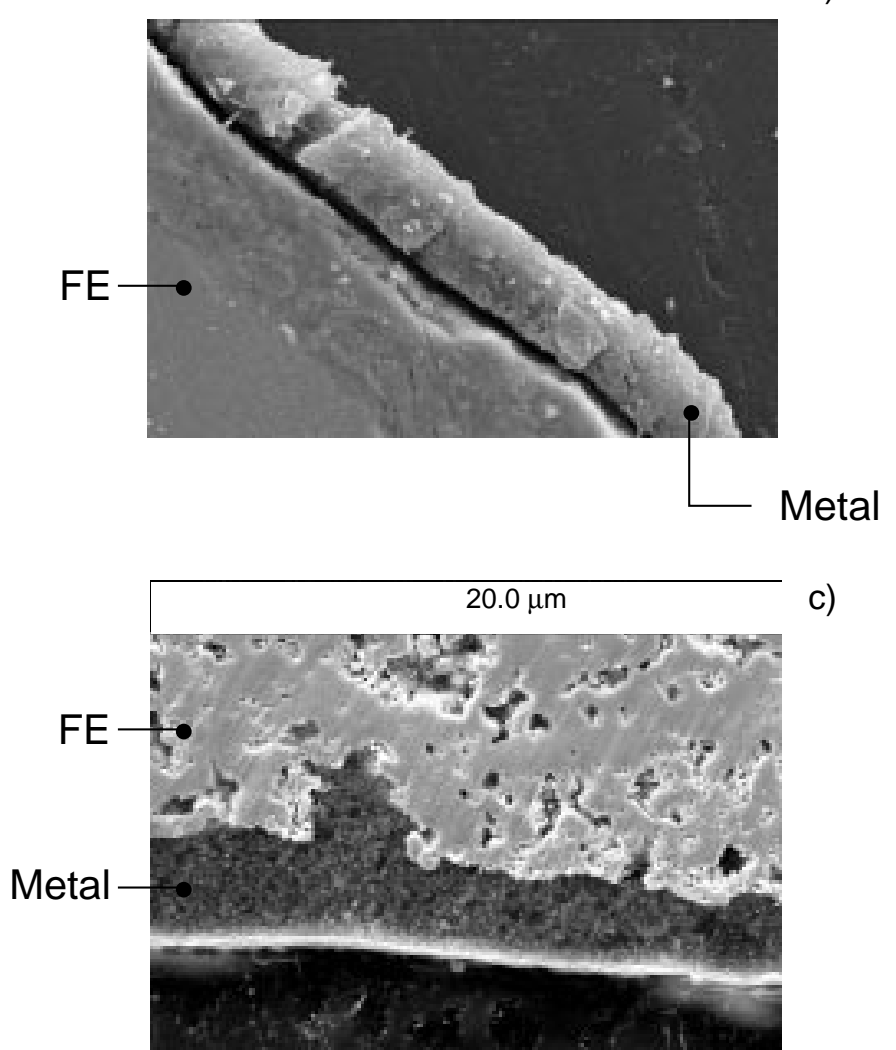

a)

\section{c)}

FIG. 13. Electron micrographs: (a) of virgin PLZT 4/95/5 material produced by the mixed-oxide method; (b) of a PLZT 4/95/5 disc cut parallel to its axis and electroded by screen-printing with an Ag-paste baked out at $600{ }^{\circ} \mathrm{C}$; (c) of a PLZT 4/95/5 cathode cut as before and covered with a low-temperature screen-printing Ag-paste (Ag L200, Degussa) fitting well to the FE surface.

\subsection{Cathode surface and electrodes}

After many years of experience with applications of FE cathodes it has been recognized that the FE cathode surface and the layout and the deposition methods of the metallic excitation electrodes count amongst the most important factors for efficiently and reliably achieving strong FE emission. If the electrical contact between the metallic grid electrodes and the bare FE surface is not quasi-perfect, FE emission cannot be excited, even with the best FE material. Other types of electron emission from FE remain not only feasible with non-ideally adhering electrodes, but may even be enhanced as is the case with reverse-field excitation [14-21] or with excitation by metal-dielectric interface breakdown [22]. The interface between electrode metal layer and the FE surface characterized by a finite roughness determines the average 
threshold voltage needed to start $\mathrm{P}_{\mathrm{s}}$-switching and FE emission. Figures 13 b) and c) demonstrate the electrode adherence to the FE surface for two different screen-printing methods: whereas the high-temperature screen-printing Ag-layer seems to make a rather bad contact (b), the low temperature Ag-paste penetrates rather well into the relief of the FE surface without forming an intermediate layer (c). Threshold field variations of more than one order of magnitude have consequently been observed with the same FE material and the same electrode material, but different electroding procedures. Also the mechanical adherence of the electrodes is strongly dependent on this interface. If the surface is too smooth there is not only little bulk field enhancement near the sharp edges of the electrodes, but the electrodes also adhere very badly to the FE surface. Similarly, the viscosity and the coagulation properties of the electroding metal may strongly influence the average $\mathrm{P}_{\mathrm{s}}$-switching fields. Whether the electrodes are deposited by sputtering, metal evaporation, screen-printing, galvanization, or painting procedures (which all imply quite differing heat treatments), different oxygen-vacancy concentrations are generated in the FE material and various oxidation processes may take place on the surface and in the adjacent layers. Heat treaments under vacuum should be avoided, since the high losses of oxygen result in a substantial de-oxygenation of the surface layers. Too high concentrations of mobile ion defects push the switching and the emission thresholds to an unacceptably high level.

Electroding has been carried out with different metals including $\mathrm{Au}, \mathrm{Ag}, \mathrm{Cu}, \mathrm{W}, \mathrm{Pb}$ and various electroding procedures. The first successful experiments with field-excited FE emission at CERN [6] were carried out with cathodes electroded under vacuum with 1- to $2-\mu \mathrm{m}$-thick Au-layers evaporated on top of a previously evaporated Cr-layer (100 $\AA$ thick). Later on other types of electroding procedure were tried out for economic reasons, but the results were less convincing. Screen-printing of excitation electrodes with metallic pastes has presented many more problems. Screen-printed FE cathodes may sometimes succeed provided good contact between electrodes and the FE surface is achieved and the FE material is not destroyed or strongly modified. The metallic electrode layers should completely fit the topology given by the roughness of the FE surface, as is the case with the above-mentioned evaporated Au-layers. With the same FE material and under equal geometrical configurations cathodes have been electroded with different types of metallic and insulating pastes, by different screenprinting methods and various heat treatments. These cathodes were found to work in a range from no emission at all to a reasonably strong emission. The main reason is an apparent variation of the average threshold voltage, sometimes rising beyond the destruction limit. The best results from screen-printing so far have been obtained with a silver paste (Ag L200 produced by Degussa) applied directly onto the FE surface without application of a Cr- layer underneath. This paste and the silicon paste used for insulating did not require any bake-out at high temperature.

If the surface was too rough or if it was strongly etched, the local field enhancement near holes, scratches or other intrusions reduced the electrical breakdown strength of the cathode, and the cathode bulk could be easily perforated by an HV switching pulse even at low average excitation-voltage amplitude. The thresholds for switching and electron emission can be lowered by heat treatments with slow cooling cycles prior to use in FE emission, by prepoling at temperatures just above the ferroelectric-paraelectric phase transition temperature, by working in a low-pressure gas or plasma atmosphere or with an FE material which is at the 
operating temperature near phase transition. A significant lowering of the threshold excitation field can be achieved by metallizing weakly the bare grid surface area with an additional nonoxidizing $(\mathrm{Au})$, thin and non-contiguous layer. During the emission phase electrons are collected at the isolated islands of the weakly evaporated layer from a large number of switched FE domains, even if their average concentration in the FE material is small. The electron emission occurs then at the sharp edges of the metallic islands by enhanced field emission. In principle this process is not real FE emission, but in practice it shows a similar behaviour.

Electron beams can be generated with cross-sections of various sizes and shapes determined by the layout of the semi-transparent grid electrodes on the GE cathode surface (Fig. 14). The size and geometry of the GE and the RE electrodes on the FE cathode, together with the cathode thickness and the dielectric constant of the FE material, determine the average field distribution in the emitting surface layer of polycrystalline materials before and after $\mathrm{P}_{\mathrm{s}}$ switching. Therefore, the electrodes must be matched to thick- [6] and thin-film FE samples [38] differently. With thin films the mutual attraction of the charge layers generated after $\mathrm{P}_{\mathrm{s}}$ switching on both surfaces of the sample must be taken into account and at a thickness of less than $10 \mu \mathrm{m}$ it is preferable to apply a modest extraction potential in order to overcome the mutual charge attraction of the charges on both sides of the film [39]. The geometry and the pressure of the HV-pulse contacts on the FE electrodes may strongly influence the local switching thresholds in the pressurized regions underneath the contacts and the surface field distribution inside the emitting grid area, before and after $\mathrm{P}_{\mathrm{s}}$-switching.

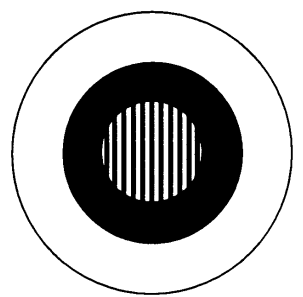

a)

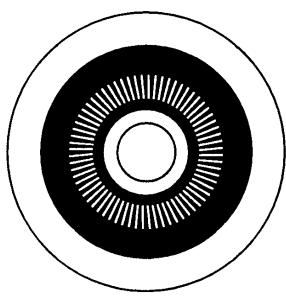

b)

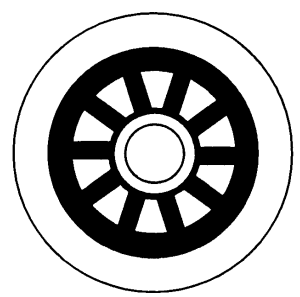

c)

FIG. 14. Axial and annular grid electrodes for axial (a), hollow (b) and multiple (c) electron beams. The inner and outer ring edges must be insulated to avoid emission from undesired surface areas.

\subsection{Environment and electrical circuits}

The parameters and the effects of the environment, inside which FE cathodes are operated merit careful analysis. The parameters of the electrical circuit for FE excitation and electron beam acceleration must be matched to the sample parameters, to the design of the vacuum gap and to the required electron beam parameters in order to guarantee a sub-microsecond HV rise time on the cathode and to avoid breakdowns through the FE cathode material or within the acceleration gaps of the electron beam transport structure adjacent to the cathode. In the past usually solid discs with a thickness of 0.1 to $1 \mathrm{~mm}$ were used as FE cathodes by the authors. Hence the pulse generator parameters had to cope with the one order of magnitude change of the cathode capacity. The same is true when changing the permittivity of the cathode material or the electrode surface area. The electrical circuits, especially the emission current loop, must be designed with low impedances in order to limit electrical power losses in stray impedances and to reach the threshold field level for $\mathrm{P}_{\mathrm{s}}$-switching of the high capacity $\mathrm{FE}$ cathode in rise times of a few tens of nanoseconds or better [7]. The excitation pulse length should not be much longer than the time taken to reach the threshold of $\mathrm{P}_{\mathrm{s}}$-switching. 
The configuration of the conductors and insulators around the vacuum gap, together with the GE of the FE cathode, determines the electrical field distribution before, during, and after switching and emission. Large, grounded metallic elements near the GE may distort the field so much that the emitted electrons cannot leave the cathode region at all. Insulated or metallic elements grounded via high impedance can exert strong focusing forces on the emitted electron beam.

In a vacuum environment the emitted electron beam current density $j_{\mathrm{e}}$ is limited by the modified Langmuir-Child law [40]

$$
j_{e}=\frac{4 \varepsilon_{0}}{9} \sqrt{\frac{2 e}{m}} \frac{1}{d^{2}}\left(\sqrt{\Delta V_{s}}+\sqrt{\Delta V_{s}+V_{e x t}}\right)^{3},
$$

where $\Delta \mathrm{V}_{\mathrm{s}}$ is the surface potential created during $\mathrm{P}_{\mathrm{s}}$-switching and $\mathrm{V}_{\text {ext }}$ the external extraction voltage. By the surface potential $\Delta \mathrm{V}_{\mathrm{s}}$ the electrons are accelerated to an initial velocity and kinetic energy, which can be further increased by applying an external extraction voltage $V_{\text {ext }}$. In absence of a plasma the gap length parameter $d$ corresponds to the width of the vacuum gap assuming plane geometry. In a realistic drift or accelerating gap containing the GE $j_{\mathrm{e}}$ is highest between bare FE surface areas and the adjacent edges of the grid electrode. The much smaller current density away from the GE towards an eventual exit aperture is fully determined by the electric field distribution inside the gap. Hence, Eq. (8) also forms the basis for the design of FE electron guns and of transport channels for space-charge dominated electron beams.

$\mathrm{P}_{\mathrm{s}}$-switching and FE electron emission were observed in vacuum, low-pressure gas, and dense plasma. In a bad vacuum environment or in a high power dissipation environment, such as in a high-power gas discharge tube, the FE cathodes may be contaminated with secondary products (e.g. rest gas is adsorbed or metal vapour deposited on the grid surface). If both effects occur on a modest level they act on the emission process favourably, as it occurs by the above-mentioned, intentionally weak metallization of the GE, provided the energy transfer and dissipation from and to the environment via the FE cathode does not deteriorate the $\mathrm{P}_{\mathrm{s}}$ switching capability. Very thin dielectric, insulating layers on the GE lead to a smear-out of the emission over several microseconds, if not to a total suppression of emission. If the FE surface is fully covered with a thick metallic layer FE emission will stop.

\subsection{Characteristics of photocathodes}

A large part of the investigations on laser-induced emission from FE cathodes covers experiments under various laser pulse conditions with FE photocathodes used in the classical way: laser illumination under a d.c. extraction field. For this 'normal' laser-induced emission, cathodes with a large bare-FE/metal ratio of the GE electrode are favourable and have been used with ratios up to $80 \%$. As described in Section II.E this scheme of employing FE photocathodes is the least interesting one and this type of electroding is unfavourable for laserinduced self-emission. The use of FE self-emission and enhancement methods makes it possible to operate FE photocathodes at infrared wavelengths and at ultra-short laser pulse lengths with high efficiency and unequalled reliability. However, the requirement for obtaining extraction field-free emission or enhancement of normal electron emission from an FE cathode is the capability of $\mathrm{P}_{\mathrm{s}}$-switching exactly in the region of the laser spot. The grid electrodes to be used for this emission mode require a bare-FE/metal ratio of $\leq 50 \%$ in the spot region, and thin 
metallic connecting rings to avoid premature switching underneath the rings compared to the emitting GE area. Most of the aspects valid for FE cathodes applied in field-excited emission, which have been discussed earlier in this section, are also valid for FE photocathodes.

The light-absorption properties of the FE material are important in the context of the conversion of photon energy into polarization energy, which results in the emission of energetic electrons. If the absorption is high (e.g. at ultraviolet wavelengths) light energy is efficiently transformed into polarization energy in a thin layer corresponding to the penetration depth. In FE material with low absorption (e.g. PLZT at visible and infrared wavelengths) the light beam penetrates a large thickness of material and produces switching with less density, but in a larger volume. Hence, the cathode thickness and the GE layout must be matched to the wavelength, energy and beam size of the laser pulse to obtain the optimum electron emission.

\section{TECHNOLOGY OF FERROELECTRIC ELECTRON GUNS}

Making use of FE self-emission and considering the limits of electron beam expansion according to Eq. (8) leads to a gun arrangement which is totally different from classical electron guns since it operates without external extraction field acting on the emitting cathode surface, possibly causing plasma formation and electrical breakdowns in the vacuum gap. The new design is based on the appearance of a negative surface charge on the bare FE surface after $\mathrm{P}_{\mathrm{s}}$ switching, generating a high electric field, under which an electron beam is expelled.

Figure 15 shows the electron gun design, which has been developed for producing medium-current electron beams to neutralize and focus ion beams generated in a laser plasma ion source of high intensity $[13,36]$. The construction is characterized by an FE sample mounted in a 20-mm-long extraction-field-free cavity of $50 \mathrm{~mm}$ inner diameter. The emitting grid electrode is grounded together with the whole cavity wall enclosing the first vacuum gap. A second, approximately 40-mm-long cavity adjacent to the first one contains a 30-mm-long focusing cylinder of $35 \mathrm{~mm}$ diameter, the field of which is screened against the first and the adjacent 10-mm-long accelerating gap. The resulting potential distribution not only reduces largely vacuum breakdown in this gap and therefore removes damaging currents and discharge plasma from the GE of the FE cathode, but also proves favourable for the transport of that part of the emitted electrons which is not lost on the grounded GE grid or on the cavity walls, towards the aperture of the high-gradient acceleration section. The electron current arriving at the entrance of the acceleration gap is given by the emitted current and the field distribution between FE cathode and acceleration gap. The field also includes the space-charge fields and consequently the limitation given by Eq. (8), when $\mathrm{e} \Delta \mathrm{V}_{\mathrm{s}}$ is replaced by the kinetic energy of the electrons at any transverse plane in the system. Figure 16a shows no influence of focusing potential on emitted current with a strongly emitting cathode contrary to Figure $16 \mathrm{~b}$ with a weakly emitting sample. Up to saturation the system behaves as a reservoir containing, after $\mathrm{P}_{\mathrm{s}}-$ switching, free energetic electrons which are extracted, when an acceleration potential is applied. Current density amplitudes of more than $10 \mathrm{~A} / \mathrm{cm}^{2}$ have been measured on the Faraday cup, exceeding the limits of Eq. (8) valid at the exit of the acceleration gap. By varying the negative potential on the focusing cylinder in the second cavity, additional flexibility is gained for intensity control by repulsion of low-energy electrons and cross-section shaping (focusing) by compressing the emitted electron beam. Magnetic focusing can also be applied before the beam reaches the accelerating gap, since the electrons leave the cathode surface with a high 
speed. The FE cathode mounted in the gun shown in Fig. 15 has a centre hole, which allows the passage of particle beams moving in the opposite direction to the emitted electron beam. Figures $17 \mathrm{a}$ and $\mathrm{b}$ show typical emitted charge and current waveforms measured with the FE electron gun shown in Fig. 15 and driven by a bipolar pulse generator as shown in Fig. 17c. The positive and the negative branch can each be pulsed with a repetition rate of up to $2 \mathrm{MHz}$. In Fig. 18 the focusing of the electron beam directed onto a luminescent screen is demonstrated with a fast CCD camera. An increase of emitted current density of one order of magnitude has been achieved by focusing with potentials of a few kilovolts. GLASS DISC

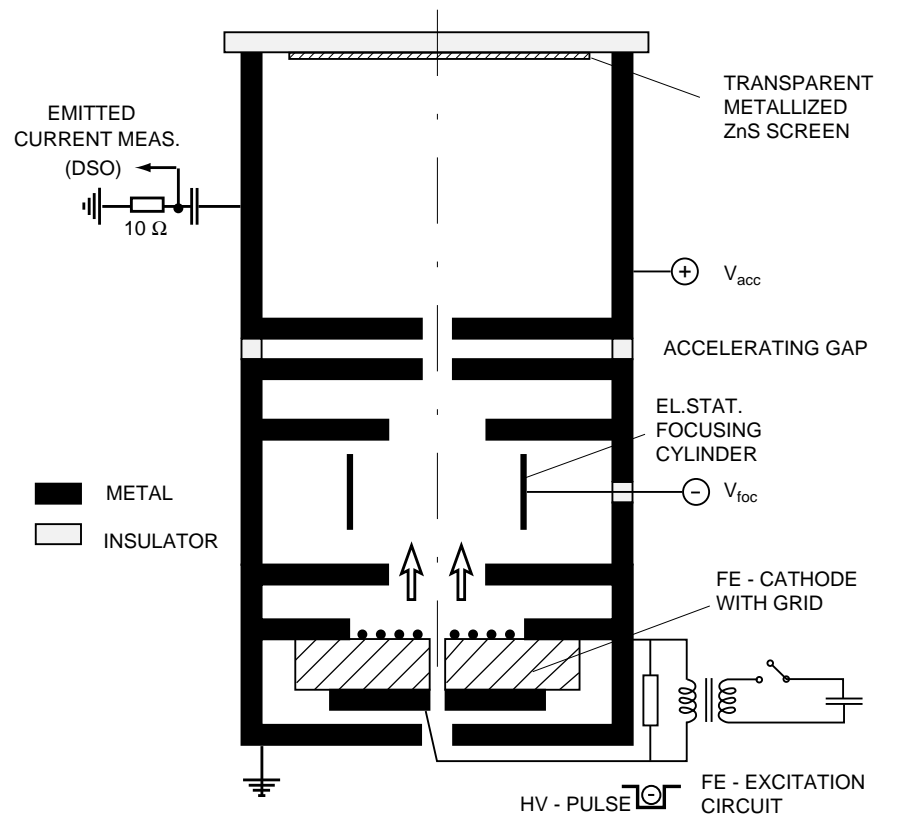

FIG. 15. Electron gun with FE cathode featuring a centre hole and zero extraction field.
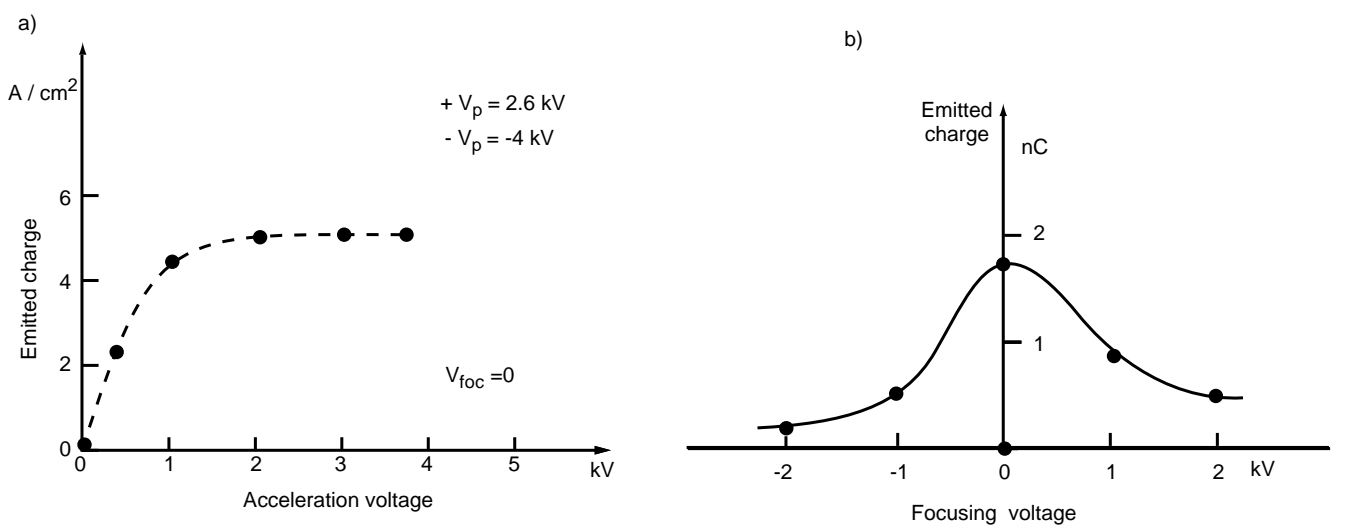

FIG. 16. a) Typical dependence of emitted current amplitude as a function of acceleration voltage for a strongly emitting PLZT 4/95/5 cathode. The voltage on the focusing cylinder has no effect on the emitted intensity. b) Case of a weakly emitting cathode showing similar behaviour with respect to accelerating potential and a clear effect of focusing potential on emitted charge at $\mathrm{V}_{\mathrm{acc}}=4 \mathrm{kV}$ from the gun shown in Fig. 15 . 


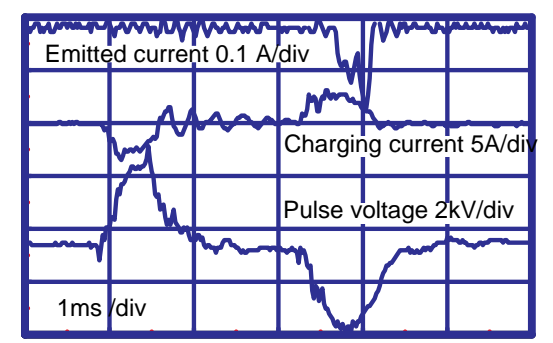

a)
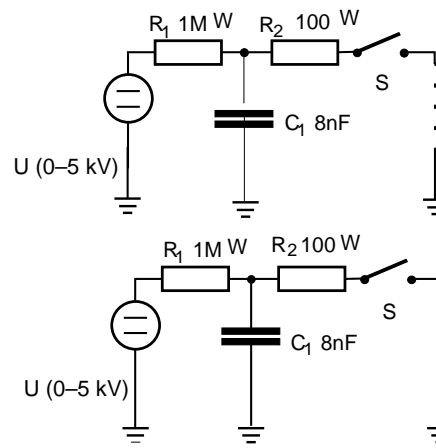

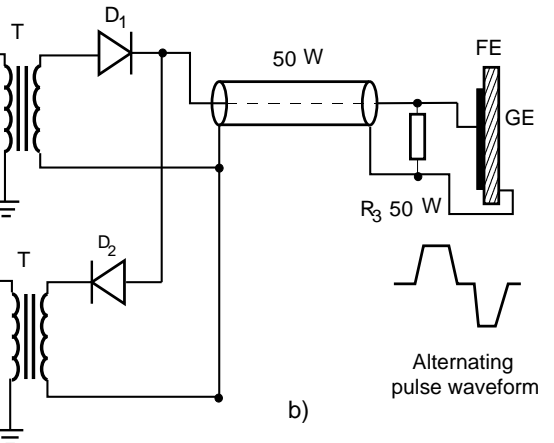

FIG. 17. Typical emitted current waveforms of electron pulses (a) generated with FE emission mode at $0.5 \mathrm{Mhz}$ repetition rate in an FE electron gun constructed as shown in Fig. 15, driven by bipolar pulse generator (b).
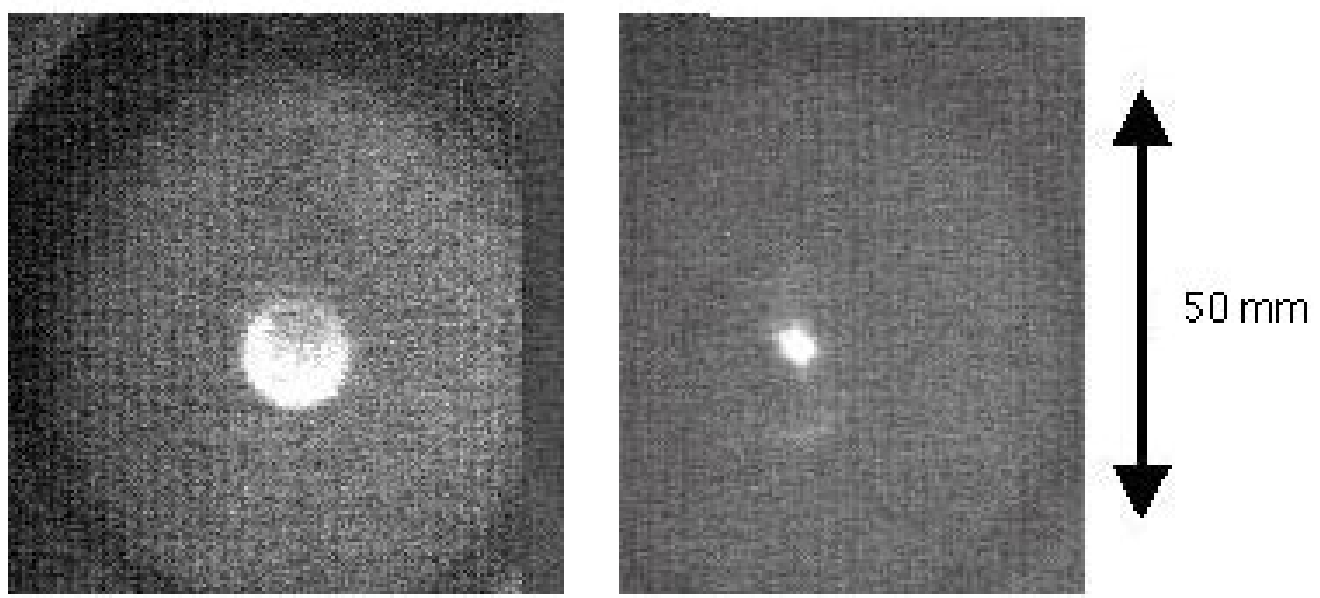

FIG. 18. Electrostatic focusing of the electron beam ejected from the FE gun by means of a focusing cylinder charged to $-2 \mathrm{kV}$ in the extraction-field-free cavity between the cathode and exit aperture and by a postaccelerating potential of $5 \mathrm{kV}$ directing the beam onto a luminescent screen.

Laser-driven electron guns with FE photocathodes can be designed in a similar way to the HV-pulse driven FE gun shown in Fig. 15. With an FE photocathode which can be efficiently switched by HV pulses in the region of the laser spot, the d.c. extraction field required for classical photoemission becomes obsolete or may be applied in a gap, separated from the FE photocathode as in the gun shown in Fig. 15. The FE photocathodes have to be excited in selfemission mode with equidistant, interleaved $\mathrm{P}_{\mathrm{s}}$-switching, preferably after each laser pulse. The post-acceleration fields should be pulsed or alternate with RF frequency, especially, if short intense beam pulses are desired and if vacuum breakdowns are to be avoided. The robustness of PLZT photocathodes has been demonstrated in experiments, where their grid-electroded surface has been illuminated with femtosecond infrared laser pulses of $0.8 \mathrm{~mJ}$ energy and $2 \mathrm{TW} / \mathrm{cm}^{2}$ peak power density at a repetition rate of $1.5 \mathrm{kHz}$ without showing any signs of destruction or fatigue [11]. Efficiency $\times$ lifetime is a performance parameter, which turns out to be very interesting for ceramic PLZT photocathodes.

\section{OTHER APPLICATIONS AND TECHNICAL PROBLEMS}

The first application of strongly emitting FE cathodes at CERN was their use as trigger elements for igniting the discharge of high-power gas switches described in Refs. [10, 11, 41]. 
The emitted beam quality and intensity were less important than high precision and robustness in the environment characterized by high power dissipation, metal plasma formation and metal vapour deposition. A more demanding application with respect to precision, repetition rate, beam quality and reproducibility is the space-charge neutralization and focusing of low-energy, heavy-ion beams described in Refs. [14, 36]. This application uses pulsed electron beams generated with an electron gun and combined with a laser ion source in a system, which includes beam diagnostic equipment for both kinds of particle beams (Fig. 19). The electron gun is constructed as shown in Fig. 15: the ion beam can traverse the electron gun and penetrate

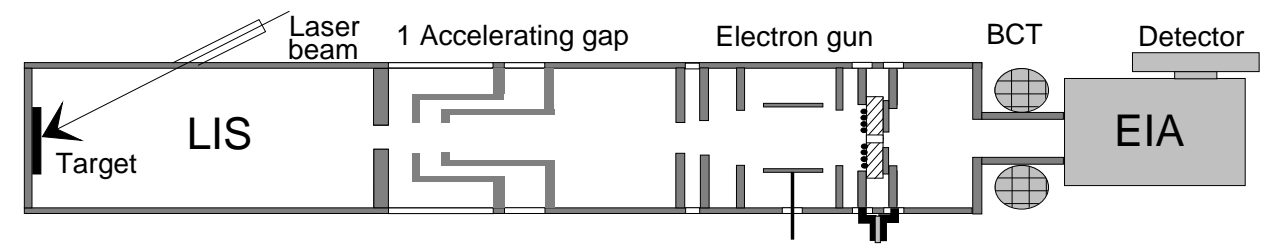

FIG. 19. FE electron gun incorporated into an ion space-charge focusing and analysing system.

into the analysing section via a hole in the centre of the FE cathode (Fig. 14b). Ion beam neutralization with ferroelectrically generated electron beams is a very promising method with applications to the intensity and brightness enhancement of a plasma ion source. While maintaining low emittance, much higher output currents can be obtained than with classical extraction methods, which are strongly space-charge limited and do not make use of the ion density potential normally contained inside such a source. Intense light- and heavy-ion sources for accelerator-based inertial confinement fusion (ICF) drivers and for neutral beam generation with application in the heating of magnetic fusion plasmas can be designed with pulsed neutralizing electron beams. Also the electrodynamic problems of high-current ion beam accelerators and around an accelerator-driven ICF target can be conveniently solved with current and space-charge neutralization by pulsed electron beams generated by guns with FE cathodes. The electron gun (Fig. 15) developed for high rep-rate, medium-intensity beams can be easily matched to other applications. With high pulsed or alternating (RF) acceleration fields short electron pulses of high intensity and arbitrary shape can be generated for klystrons and injectors for electron accelerators. Thin-layer FE emission was proposed in 1989 [38] for microwave generation and ultraflat panel TV screens. Development work performed in other laboratories has been described, for example in Refs. [16, 33, 39]. FE self-emission from thinfilms functions with very low excitation voltage amplitudes $(<100 \mathrm{~V})$, but only above a minimum FE layer thickness, which is determined by the mutual attraction of the separated charge layers formed on the opposite sides of the FE thin-film cathode. For thinner layers extraction potentials must be applied [39]. Many techniques useful for the production and preparation of thin-layer FE cathodes are available from electronic thin-film and FE memory technologies as well as from high- $\mathrm{T}_{\mathrm{c}}$ superconductor technology. The powder-type cathode described in Section III.A is especially suited for large-area emission applications, such as high current, large gas-volume irradiation and ionization (e.g. exhaust gas transmutation or other chemical reaction initialization). FE powder cathodes may also be employed in electron guns with cathodes featuring non-planar, bent emitting surfaces for any purpose, such as tubes and cones. Shaped (e.g. conical) cathodes of this type may be operated to focus electron beams simply by the cathode geometry without the use of electric or magnetic focusing fields. 


\section{CONCLUSION}

Based on the experiences of several years of development strong FE emission has been defined and described as the most promising way to cover a large range of applications. Other methods of electron generation by ferroelectrics, which look similar at first glance, differ from FE emission in terms of physical phenomena, which turned out to be major obstacles in specific applications.

The usefulness of FE electron emission has been shown for the efficient triggering of high-power gas switches, in high-intensity, high-repetition rate, HV-pulse-driven electron guns with an extraction-field-free emitting surface, for FE photocathodes featuring self-emission and outstanding robustness, for cheap FE powder electrodes and in FE thin-layer emission.

Since the discovery of strong FE emission many technological advances have been made in the frame of $R \& D$ on a few specific applications. The mixed-oxide production method of PZT and PLZT ceramics from very pure metal oxides has proven especially successful for FE emission. The decisive influence on cathode performance of the interface between the FE cathode surface and the metallic excitation electrodes has been demonstrated with different electroding procedures and electrode metals. Low-impedance circuits are required for the excitation and emission current loops. Contrary to classical emission an environment of lowpressure gas or plasma does not disturb FE cathodes and photocathodes, but enhances significantly the FE emission. FE emission allowed electron guns to be designed with an extraction-field-free cathode arrangement, generating intense $\left(>10 \mathrm{~A} / \mathrm{cm}^{2}\right)$ beam pulses with $>2 \mathrm{MHz}$ repetition rate and 50 to $200 \mathrm{~ns}$ pulse length, which can be easily electrostatically focused. Much more development work is needed to push the technological limits further and to optimize the performance of FE cathodes and guns in arbitrary applications.

For the future a large range of additional applications can be identified, such as machining applications, electron guns for low- and high-energy accelerator technology, thin-layer applications (ultra-flat TV screens, microlasers), efficient photocathodes at IR wavelengths, power tubes for RF, microwave and X-ray sources for medical applications and food sterilization, and large volume ionization for exhaust gas transmutation and chemical reaction initialization. More R\&D is required for specific applications in order to systematically optimize performance and to solve the technological and material problems partly mentioned in this paper. However, the starting conditions for this R\&D are promising, as a great deal of the technological know-how gained so far can be used from previous experiences, e.g. from the fields of electronics, classical ferroelectrics, thin-film and high- $\mathrm{T}_{\mathrm{c}}$ superconductor technologies.

\section{Acknowledgements}

The authors are indebted to J. Dalin and S. Sgobba for the electron-microscopic investigations of various FE cathodes and to E. Riedo for the systematic tests of several series of FE cathodes. To the HP group of the CERN PS Division we are grateful for the temporary loan of a CCD camera. 


\section{References}

[1] R.C. Miller, S. Savage, J. Appl. Phys. 21 (1960) 662.

[2] R. Rosenblum, P. Bräunlich, J. Carrico, Appl. Phys. Lett. 25, 17 (1974).

[3] G. Rosenman, V. Okhapkin, Yu. Chepelev and V. Shur, Sov. Phys. -JETF's Letts., (USA) 39 (1984) 477.

[4] H. Riege, H. Gundel, J. Handerek, E.J.N. Wilson, and K. Zioutas, Paper presented at the XIIIth Int. Symp. on Discharges and Electrical Insulation in Vacuum, Paris, 28 June, 1988, published in IEEE Trans. Electr. Insulation 25 (1990) 655.

[5] H. Gundel, H. Riege, E.J.N. Wilson, J. Handerek, and K. Zioutas, Nucl. Instrum. Methods A280 (1989) 1.

[6] H. Gundel, H. Riege, E.J.N. Wilson, J. Handerek, and K. Zioutas, Ferroelectrics 100 (1989) 1.

[7] H. Gundel, J. Handerek, and H. Riege, J. Appl. Phys. 69 (1991) 975.

[8] J. Handerek and H. Riege, Ferroelectrics 128 (1992) 43.

[9] K. Geissler, A. Meineke, H. Riege, and J. Handerek, Nucl. Instrum. Methods A340 (1994) 96.

[10] H. Riege, Nucl. Instrum. Methods A340 (1994) 80.

[11] H. Riege, P. Faure, K. Schmidt, G. Schröder, J. Handerek, P. Benin, L. Courtois, Digest of 9th IEEE Int. Pulsed Power Conf., Albuquerque, NM (1993) 364.

[12] K. Geissler, A. Meineke, H. Riege, S. de Silvestri, M. Nisoli, O. Svelto, I. Boscolo, J. Handerek, Nucl. Instrum. Methods A372 (1996) 567.

[13] U. Herleb, H. Riege, Proc. of the 11th Int. Conf. on High Power Particle Beams, Prague, edited by K. Jungwirth and J. Ullschmied, Vol. I (1996) 68.

[14] J.D. Yvers, L. Schachter, J.A. Nation, G.S. Kerslick and R. Advani, J. Appl. Phys. 73 (1993) 2667.

[15] L. Schachter, J.D. Yvers, J.A. Nation, and, G.S. Kerslick, J. Appl. Phys. 73 (1993) 8097.

[16] J. Asano, T. Imai, M. Okuyama, Y. Hamakwa, Jpn. J. Appl. Phys. 31 (1992) 3098.

[17] S.E. Sampayan, G.J. Caporoso, C.L. Holmes, E.J. Lauer, D. Prosnitz, G. Westenskow, Nucl. Instrum. Methods A340 (1994) 90.

[18] S.E. Sampayan, G.J. Caporoso, D. Trimble, G. Westenskow, Proc. Particle Accelerator Conf., Dallas, 1995, vol. 2 (1995) 899.

[19] T.C. Cavazos, W.L. Wilbanks, C. Fledderman, D.A. Shiffler, Appl. Phys. Lett. 65 (1994) 2612.

[20] A. Airapetov, I. Ivanchic, A. Lebedev, I. Levshin, and N. Tikhomirova, Sov. Phys. Dokl. 35 (1990) 267.

[21] G. Benedek, I. Boscolo, J. Handerek, H. Riege, J. Appl. Phys. 81 (1997) 1396.

[22] V.F. Puchkarev, G.A. Mesyats, J. Appl. Phys. 78 (1995) 5633.

[23] G. Rosenman, V.I. Pechorskii, and I. Rez, Sov. Phys. Solid State 23 (1981) 2162. 
[24] G. Rosenman, V.I. Pechorskii, Y. Chepelev, E. Boikova, L. Isakova, Phys. Status Solidi B120 (1983) 667.

[25] G. Rosenman and I. Rez, J. Appl. Phys. 73 (1993) 1904.

[26] K. Biedrzycki, Z. Czapla, D. Averty, H. Aboura and R. Le Bihan, Ferroelectrics 155 (1994) 135.

[27] G. Benedek, I. Boscolo, J. Handerek, H. Riege, Nucl. Instrum. Methods A393 (1997) 469.

[28] H. Gundel, H. Riege, E.J.N. Wilson, J. Handerek, and K. Zioutas, Ferroelectrics 94 (1989) 337 and Preprint CERN PS/88-54 (AR).

[29] J. Handerek, Z. Ujma, C. Carabatos-Nédelec, G.E. Kugel, D. Dmytrow, I. El-Harrad, J. Appl. Phys. 73 (1993) 367.

[30] K. Geissler, A. Meineke, H. Riege, J. Handerek, H. Granzer, and D. Suchland, Phys. Lett. A166 (1992) 84 and Preprint CERN/PS 92-27 (AR).

[31] A. Meineke, Doctoral Thesis, Tech. University of Berlin, 1995.

[32] D. Shur, G. Rosenman, Y. Kraslk and V. Kugel, J. Appl. Phys. 79 (1996) 3669.

[33] G. Rosenman, D. Shur, and A. Skliar, J. Appl. Phys. 79 (1996) 7401.

[34] Brevet d'invention FR 2 639151, Nº de Registr. 8808636, INPI, Paris (1988).

[35] K. Geissler, J.P. Gourber, A. Meineke, H. Riege, B. Leblond and J. Handerek, Paper presented at the Fourth EPAC, London, UK, June 1994 and Preprint CERN AT/94-34 (DI) (1994).

[36] U. Herleb, H. Riege, Proc. of the 11th Int. Conf. on High Power Particle Beams, Prague, edited by K. Jungwirth and J. Ullschmied, Vol II (1996) 1191.

[37] Brevet d'invention FR 2718567 - B1, Nº de Registr. 9404424, INPI, Paris (1994).

[38] European Patent, EU 90119196.5, Prior. DE 3938752, 23.11.1989, Publ. Nr. 0 428853 B1 (1991).

[39] D. Averty, S.F. Liateni, R. Le Bihan, Ferroelectrics 173 (1995) 171.

[40] H. Rothe, W. Kleen, Elektronenröhren als End- und Sendeverstärker, Leipzig, Akad. Verl.-Ges. (1940).

[41] L. Ducimetiere, P. Faure, U. Jansson, H. Riege, M. Schlaug, G. Schröder, E.Vossenberg, Paper presented at the 22nd Int. Power Mod. Symposium, Boca Raton, June 1996. 